\title{
Review of Helicon High-Density Plasma: Production Mechanism and Plasma/Wave Characteristics
}

\author{
Shogo ISAYAMA, Shunjiro SHINOHARA ${ }^{1)}$ and Tohru HADA ${ }^{2)}$ \\ Department of Physics, National Central University, Taoyuan 32001, Taiwan \\ ${ }^{1)}$ Institute of Engineering, Tokyo University of Agriculture and Technology, 2-24-16 Naka-cho, Koganei, \\ Tokyo 184-8588, Japan \\ ${ }^{2)}$ Interdisciplinary Graduate School of Engineering Sciences, Kyushu University, 6-1 Kasuga-Kohen, Kasuga, \\ Fukuoka 816-8580, Japan
}

(Received 26 December 2017 / Accepted 8 February 2018)

\begin{abstract}
Helicon plasma is one of the radio frequency plasma source that can generate high-density and lowtemperature plasmas by utilizing the helicon wave, i.e., the electromagnetic whistler wave in a bounded geometry. Helicon plasma is very useful for various applications due to its extremely efficient production of high-density plasma. Conversely, many unsolved physical issues remain regarding how an efficient production of the helicon plasma is realized in laboratories and what determines the density maximum. The past decades of the helicon studies have revealed that the "Trivelpiece-Gould" wave is responsible for the efficient power absorption. In recent years, the drift-wave type and the parametric-decay instabilities have been extensively studied, by using the linear magnetized helicon plasma sources. The present helicon study considers these non-linear effects. Consequently, it includes many interests for both industry and research fields. The mechanism of the helicon production is discussed, based on the several critical physical issues. In addition, some recent topics and the efforts to build a more refined physical model of the helicon plasma are highlighted.
\end{abstract}

(c) 2018 The Japan Society of Plasma Science and Nuclear Fusion Research

Keywords: helicon plasma, helicon wave, TG wave, neutral depletion, drift-wave instability, parametric-decay instability

DOI: $10.1585 /$ pfr.13.1101014

\section{Introduction}

Helicon plasma sources attract keen interest worldwide. These types of plasma sources can generate a uniform, high-density (electron density up to $10^{20} \mathrm{~m}^{-3}$ ) and low-temperature (electron temperature from a few to several eV) plasma, under a wide range of parameters, e.g., device scale, magnetic field, neutral pressure [1-4]. Due to these distinctive advantages, the helicon source is used for various applications including plasma processing, nuclear fusion, electric thrusters, and space laboratory experiments [5-7].

The helicon plasma is generated by using the helicon wave, i.e., electromagnetic whistler wave in a bounded plasma. Over the past decades, research on the helicon plasma has revealed some important mechanisms of efficient plasma production. One part of the efficient power absorption can be explained by the strong collisional damping of the quasi-electrostatic wave or so called "Trivelpiece-Gould" (TG) wave [8]. The TG wave is excited dominantly near the radio frequency (RF) antenna and deposits its energy near the plasma surface [9-11], while the helicon wave penetrates into the core region and deposits its energy via collisions in the core plasma $[9,12,13]$. The existence of the TG wave is experimentally

author's e-mail: isayamashogo@gmail.com verified [14], and the dispersion relation and the analysis of the power balance explain, at least qualitatively, some distinct features observed in the helicon discharge.

Contrariwise, recent studies suggested that not only the directly excited waves but also some non-linear parametrically excited waves can contribute to the power absorption and ion heating [15-17]. In addition, the driftwave type instabilities have also been observed in the large linear helicon devices [18-21]. Drift-waves can cause the anomalous plasma transport across the magnetic field [22], which result in notable losses of particles and energy from the confinement regions by influencing the equilibrium states of the particles and the power balances [18]. Therefore, the present helicon studies need to consider these non-linear effects on the plasma transport and discharge equilibrium. Furthermore, for developing the RF plasma sources with higher efficiency and high density, it is inevitable to clarify the mechanism of the neutral depletion [23], which is thought to limit the maximum density of the helicon source [5, 24, 25].

This review intends to describe a basic understanding of the helicon plasma production mechanism, and highlight some recent topics and remaining critical issues. In Sec. 2, a brief history of the helicon study is overviewed. Section 3, describes a simple model of the helicon wave, 
with its dispersion relation and experimental results. The role of the electron inertia, which adds the second dispersion of the TG wave, is then discussed (Sec.4). Section 5 examines the resonance and anti-resonance properties of the excited waves in the cylindrical bounded system, which leads to the non-linear power absorption curves as a function of the plasma density or the magnetic field. In Sec. 6, one of the distinct features of the density jumps observed in the experiments are discussed, based on the non-linear power absorption curves, and the unsolved issue is also suggested. Section 7 describes the experimental and theoretical studies of neutral depletion. In Sec. 8, one of the controversial issues of the plasma transport in the RF sources are overviewed. Recent studies about the instabilities in the helicon source (Sec. 9) and other notable experiments (Sec. 10) are reviewed. In Sec. 11, the remaining critical issues are summarized and attempts to clarify these matters by the self-consistent model are suggested in Sec. 12. In Sec. 13, the applications of the helicon source are introduced. Finally, Sec. 14 provides some concluding remarks.

\section{History of the Helicon Study}

The term "helicon" was named by Aigrain in 1960 [26], to describe the whistler wave propagating in solid metal. The helicon wave in metallic sodium was first observed by Bowers, Legendy, and Rose [27], who also investigated its dispersion relation. The linear relationship between the frequency and the strength of the static magnetic field, observed in Kanai's [28] experiment, showed good agreement with the theoretical prediction. In 1965, Lehane and Thonemann [29] conducted the first study of helicon wave propagation in a gaseous plasma. The phase velocity, the structure, and the decay length of the helicon wave corroborated the theory proposed by Klosenberg, McNamara, and Thonemann [30].

In 1970, Boswell [31] discovered that high-density plasma can be generated by exciting helicon waves. Boswell et al. [1,32] investigated the structures of the helicon waves with theoretical fittings and the average electron density variation due to the magnetic field and gas pressure. In Boswell's experiments, however, it was found that the effective collision frequency had to be at least 1,000 times greater than the theoretical collision rate to explain the observed efficient ionization and radial wave profiles. In 1991, Chen suggested that Landau damping could be the cause of the efficient energy absorption [2,33]. This mechanism was adopted by authors, such as [34,35] and supported by the detection of fast electrons by Ellingboe $e t$ al. [36] and Molvik et al. [37] using RF-modulated emissions of $\mathrm{Ar}^{+}$light. However, by using an energy analyzer that accounts for RF fluctuations in plasma potential, Chen and Blackwell [38] quantitatively demonstrated that the number of fast electrons was insufficient to explain the high efficient ionization. Thus, the Landau damping for efficient ionization was considered unimportant.

Shamrai et al. [11,39-43] suggest that the strong collisional damping of the TG wave can explain the effective power absorption. In the following frequency regime,

$$
\omega_{\mathrm{ci}} \ll \omega_{\mathrm{LH}}<\omega \ll \omega_{\mathrm{ce}} \ll \omega_{\mathrm{pe}},
$$

where $\omega$ is the RF driving frequency, $\omega_{\text {pe }}$ and $\omega_{\text {ce(ci) }}$ are the plasma and the gyro-frequencies of electrons (ions), respectively, and $\omega_{\mathrm{LH}}$ is the lower hybrid frequency. The dispersion relation of magnetized cold plasma can be written as [42],

$$
k_{\perp \pm}^{2}=\frac{k_{\|}^{2}}{2 \gamma^{2} \alpha^{2} \beta^{2}}\left(1-2 \gamma \alpha-2 \gamma^{2} \alpha^{2} \beta^{2} \pm \sqrt{1-4 \gamma \alpha}\right),
$$

where $k_{\perp}$ and $k_{\|}$are the perpendicular and parallel wavenumbers in the background magnetic field, respectively, and

$$
\begin{aligned}
& \alpha=\frac{\omega_{\mathrm{pe}}^{2}}{\omega_{\mathrm{ce}}^{2} N^{2}}, \beta=\frac{\omega \omega_{\mathrm{ce}} N^{2}}{\omega_{\mathrm{pe}}^{2}}, \gamma=1+i\left(\frac{\nu}{\omega}\right), \\
& N=\frac{c k_{\|}}{\omega},
\end{aligned}
$$

where $c$ is the speed of light and $v$ is the electron collision frequency between the neutrals and ions. Two different wave modes exist; the negative and the positive signs correspond to the helicon wave and the TG wave, respectively. Considering the density profile shown in Fig. 1 [43], one may note the three-characteristic density regions. The first is the low-density region ( $n<n_{\text {low }}$ and $r>r_{\text {low }}$ ), where only the TG is propagating while the helicon wave is nonpropagating; the second is the intermediate-density region ( $n_{\text {low }}<n<n_{\text {up }}$ and $\left.r_{\text {up }}<r<r_{\text {low }}\right)$, where both the helicon and the TG waves are propagating; and the third is the high-density region ( $n>n_{\text {up }}$ and $\left.r<r_{\text {up }}\right)$ where both waves become evanescent. In the typical experimental

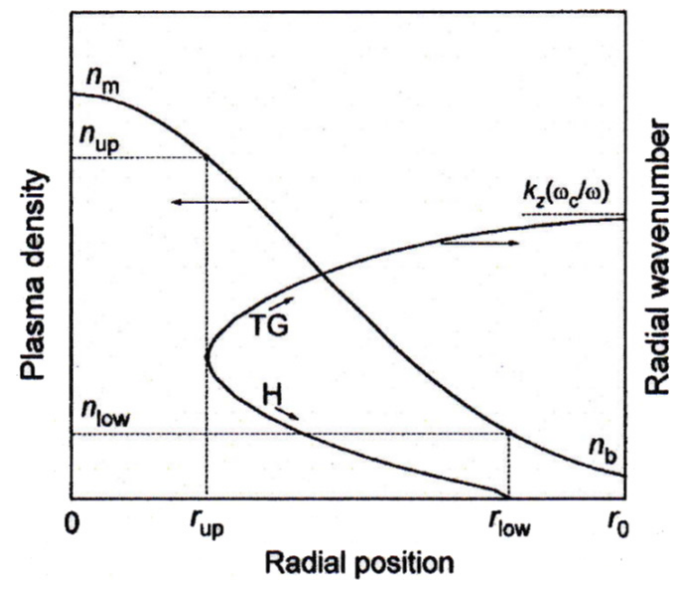

Fig. 1 The density profile and the radial variation of transverse wavenumber in the local approximation. Helicon $(\mathrm{H})$ and Trivelpiece-Gould (TG) branches of the dispersion curve [43]. 
conditions $[1,44,45]$, the damping length of the TG wave is much shorter than for the plasma column $k_{\perp+\text { imag }} r_{0} \gg 1$, where $k_{\perp+, \text { imag }}$ is the imaginary part of the $k_{\perp+}$, and $r_{0}$ is the plasma radius, while that of the helicon wave is longer than the plasma column $k_{\perp+\text {,imag }} r_{0}<1$ except for in the highdensity region $\left(n \sim n_{\text {up }}\right)$, where the damping length of the helicon wave becomes comparable to that of the TG wave. Therefore, the collisional damping of the TG wave plays a dominant role on the surface power absorption while the helicon wave is important for power absorption in the central high-density region $[9,12,13,46]$.

The quasi-electrostatic TG wave is excited by two mode conversion mechanisms [43]. The first is the mode conversion that occurs at the plasma surface. Since the radial current $j_{r} \approx E_{r}+i\left(\omega / \omega_{\mathrm{ce}}\right) E_{\theta}$ must vanish at the plasma surface due to the boundary condition, the strong radial electric field $E_{r}$ is generated to balance $\left(\omega / \omega_{\mathrm{ce}}\right) E_{\theta}$, and the TG wave is consequently excited. The second is the bulk mode conversion, which occurs near the mode conversion surface (MCS) where the helicon and the TG wave branches merge at $n=n_{\text {up }}$ as shown in Fig. 1 . The helicon wave penetrates into the high-density region due to its weak damping rate, and when the helicon wave reaches $n=n_{\text {up }}$, it is reflected and naturally transformed to the backward propagating TG wave from this merging point. However, when a finite collision is introduced, the efficiency of the bulk mode conversion becomes strongly dependent on the magnitude of the collision frequency [13].

The range of the plasma density produced by the helicon source is $n \sim 10^{16}-10^{20} \mathrm{~m}^{-3}[47,48]$. There are believed to be there are three distinct modes of operation for a helicon plasma source: the capacitively coupled plasma (CCP or E) mode [49-51], the inductively coupled plasma (ICP or $\mathrm{H}$ ) mode $[52,53]$, and the helicon wavesustained (W) mode. The E-H-W mode transitions are observed with jumps in the plasma density. Transitions between the low- and high-density modes are shown by varying the external magnetic field $[1,44,54]$, the RF input power [44, 47, 55-59], and the neutral pressure [58]. The E-H transition occurs at a density of approximately $10^{16}-10^{17} \mathrm{~m}^{-3}[48,52,60,61]$. This mode transition generally occurs when the collisionless skin depth $\delta=c / \omega_{\text {pe }}$ is equal to approximately half a plasma radius [48]. The $\mathrm{H}-\mathrm{W}$ transition occurs at a density of approximately $10^{17}$ $10^{18} \mathrm{~m}^{-3}$ [48]. This mode transition is characterized by the dispersion relation of the helicon wave [47, 48, 57, 59]. Before the density jump, the wave fields are localized near the antenna with a standing wave character, and the dispersion relation of the helicon wave is not satisfied. After the jump, the propagating waves are excited and the dispersion relation of the helicon wave is confirmed.

In recent studies, Kline and Scime [16] suggest a nonlinear coupling mechanism between the helicon wave and the electrostatic waves. They showed that the helicon pump wave can decay into the ion-acoustic wave and the TG wave through parametric decay instability. Altukhov et al. [62] first evidenced this mechanism by the spectral measurements. Krämer et al. [17,63,64] presented the threshold of the pump field for this instability, and the growth rates of the parametric instability, which fit the experimental results reasonably well.

As one of the eminent characteristics of helicon discharge, it is well known that plasma density increases with the magnetic field strength in an essentially linear fashion [44]. This linear relationship is derived from the simple theory of the bounded helicon wave dispersion relation with fixed $k_{\|}$[2]. The observed non-monotonic density jumps with increases in the magnetic field that arise from the mode transition among different axial wave modes $k_{\|}$ $[1,65]$. However, many experiments have observed an unexpected decrease in the plasma density with increases in the magnetic field strength [55,66-69]. This may be explained using the framework of low-frequency electrostatic instability, which can cause anomalous radial diffusion and greatly affect the plasma equilibrium [18]. The candidates to drive such diffusion are the resistive drift-wave instability [70-75] driven by the gradient of the plasma density and the Kelvin-Helmholtz [76-79] or the Simon-Hoh instabilities $[80,81]$ driven by the gradient of the spatial potential. Light et al. $[18,82]$ experimentally demonstrated good agreement of the theory and verified the existence of such instabilities.

\section{Simple Model of the Helicon Wave}

The helicon wave is essentially a bounded whistler wave propagating in a dc magnetic field, and the dispersion relation of the helicon wave is derived from the standard theory of whistler waves in cold plasma. In the basic model of the helicon wave, the electron inertia term is neglected in the limit of zero electron mass $m_{\mathrm{e}}=0$, and the axial electric field $E_{z}$ also vanishes [2,83]. The dispersion relation is given at the frequency range of (1) as follows:

$$
\frac{c^{2} k^{2}}{\omega^{2}}=\frac{\omega_{\mathrm{pe}}^{2}}{\omega \omega_{\mathrm{ce}} \cos \theta},
$$

where $k=\left(k_{\perp}^{2}+k_{\|}^{2}\right)^{1 / 2}$ is the wavenumber, $\theta$ is the angle of wave propagation relative to $B_{0}$, and $k_{\|}=k \cos \theta$ is the parallel (axial) wavenumber. The dispersion relation above (4) may be written as

$$
k=\frac{\omega}{k_{\|}} \frac{\omega_{\mathrm{pe}}^{2}}{\omega_{\mathrm{ce}} c^{2}}=\frac{\omega}{k_{\|}} \frac{n_{0} e \mu_{0}}{B_{0}},
$$

where $e$ and $\mu_{0}$ are the electric charge and the permittivity of free space, respectively. When the wave is confined to a cylinder of radius $a$, for cases with an insulating or conducting boundary at $r=a$, the boundary conditions for the waves with $k_{\|} a \ll 1$ are derived from the condition of $B_{r}=0$ as

$$
m k J_{m}\left(k_{\perp} a\right)=-k_{\|} a J^{\prime}\left(k_{\perp} a\right) \sim 0 \quad\left(k_{\|} a \ll 1\right),
$$

where $J_{m}$ is the $m^{\text {th }}$ order Bessel's function. For $m=1$, the lowest Bessel root is $k_{\perp} a=3.83$, and therefore, for $k_{\|} \ll$ 


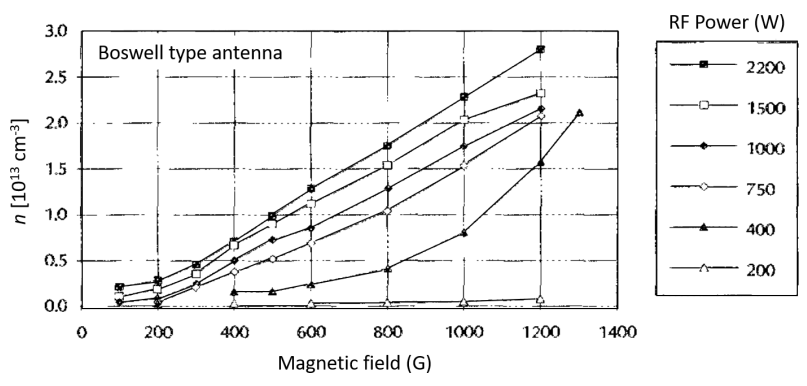

Fig. 2 Linear relationship between the plasma density and the magnetic field [44].

$k_{\perp}$, the dispersion relation can be approximately obtained as [86]

$$
\frac{3.83}{a}=\frac{\omega}{k_{\|}} \frac{n_{0} e \mu_{0}}{B_{0}} \propto \frac{\omega}{k_{\|}} \frac{n_{0}}{B_{0}} .
$$

This shows the linear relationship for a given mode $(\omega$ and $k_{\|}$are fixed) between the density $n$ and the magnetic field $B_{0}$. Figure 2 [44] shows the $n-B$ curve as the RF power varies with the Boswell-type antenna [35]. When $P_{\mathrm{RF}}>400-500 \mathrm{~W}$ and $B_{0}>400-500 \mathrm{G}$, the value of $n / B$ is approximately constant, as expected from (7). The basic $n-B$ relationship above threshold values of $P_{\mathrm{RF}}$ and $B_{0}$ has been verified by a number of authors such as Boswell, Porteus, and Ellingboe [47,84], who suggest that a certain threshold is related to the discharge mode transition from the ICP to the helicon plasma. When the magnetic field is weak and RF power is low in relation to the ICP operation, the density is not high enough to satisfy (7). If $P_{\mathrm{RF}}$ or $B_{0}$ is raised, the helicon wave is excited and (7) is satisfied [85]. Chen et al. [86,87] also calculated the wave magnetic field with the measured density profile, and showed good agreements with the $m=1$ mode radial wave profiles as expected for the right-hand polarized antenna. Shinohara et al. [88] also found agreement for the radial wave profiles of the $m=0$ helicon mode when excited by a planar spiral antenna.

\section{Role of the Electron Inertia}

Davis [89] pointed out that, even when the wave frequency is much lower than the electron cyclotron frequency, the electron inertial term must be accounted for in the generalized Ohm's law [90]. Boswell [91] first investigated the effects of the electron inertia on the dispersion relation of helicon waves. The TG wave strongly dissipates at the collision frequencies typical for the experiments and deposits its energy in a narrow surface layer, while the helicon waves penetrate the bulk plasma [11,41-43]. The numerical calculations by Mouzouris and Scharer [9], Park et al. [10], and Shamrai and Shinohara [11] showed that the major RF power is deposited by the TG wave at the plasma surface.

Lho et al. [92] detected the TG wave in the low-

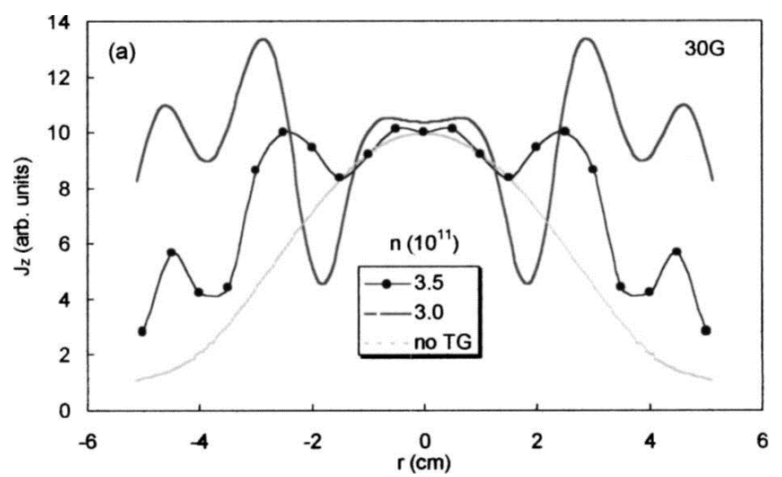

Fig. 3 Radial profiles of RF current $\left|J_{z}\right|$ at $30 \mathrm{G}$ as measured (dot-solid line), as predicted by theory (solid line), and as calculated from simple helicon theory without the TG mode (dotted line) [14].

magnetic field $\left(B_{0} \sim 16-24 \mathrm{G}\right)$ helicon discharge and showed the observed dispersion relation of the TG wave with theoretical fittings. The radial structures of the TG wave were clearly detected by Blackwell et al. [14] in an experiment with a low magnetic field $\left(B_{0}<50 \mathrm{G}\right)$. The wavelength of the TG wave becomes comparable to that of the helicon wave with such a low magnetic field, and such as, the TG wave structure extends far into the interior of the plasma and its effect on the wave shapes is detectable. Figure 3 shows the $\left|J_{z}\right|$ profiles measured at $B_{0}=30 \mathrm{G}$ and $n=3.5 \times 10^{11} \mathrm{~cm}^{-3}$, compared to curves calculated by the HELIC code [12,93] (at the density $n=3.0 \times 10^{11} \mathrm{~cm}^{-3}$ ) with and without inclusion of the TG wave. This figure has "wings" structures due to the presence of the TG waves around the plasma surface regions $(|r|>2)$, that are not present with the helicon wave alone.

The TG wave is excited at the plasma surface (surface mode conversion) and in the bulk high-density region of the plasma (bulk mode conversion). The bulk mode conversion occurs near the MCS. Based on the dispersion relation, Kim and Hwang [94] pointed out that for bulk mode conversion, the collisional dissipation near the MCS plays an important role. Isayama et al. [13] report the quantitative dependence of the bulk mode conversion efficiency on the collision frequency. Figures 4 (a) and (b) show the spatial variations of the real wavenumber $k_{\perp \text { real }}$ and the normalized imaginary wavenumber $k_{\perp \text { imag }} x_{0}$ (where $x_{0}=$ $7.5 \mathrm{~cm}$ is the plasma size) of the helicon wave (red line) and the TG wave (blue line), respectively. Here, the parabolic density profile, which peaks at $x=0$, is assumed to have the various values of the collision frequency $v / \omega=0$ (solid line), 0.01 (dashed line), and 0.08 (dotted line). When the collision is absent, the dispersion curves of the helicon and the TG wave merge at $x=x_{\text {up }}$, corresponding to the MCS where the discriminant in (2) is zero. In this case, a helicon wave propagating toward the negative direction converts to a TG wave as a reflected wave (the group velocity is in the positive direction). When a finite collision 

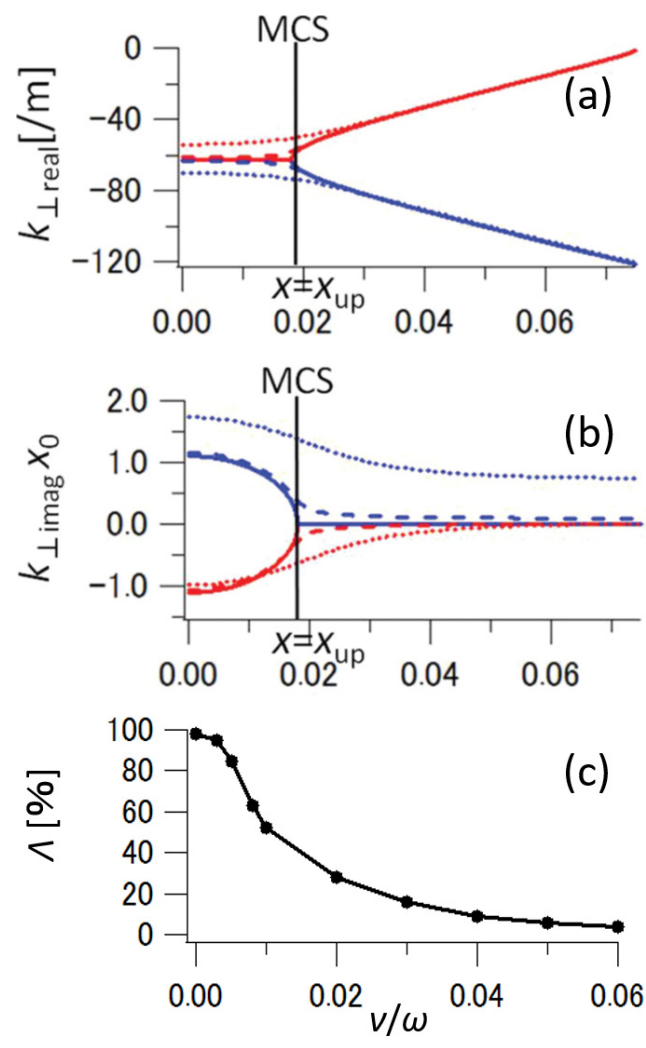

Fig. 4 (a) Real and (b) normalized imaginary parts of transverse wavenumbers of the helicon (red) and TG waves (blue) with $v / \omega(\omega / 2 \pi=7 \mathrm{MHz})=0$ (solid), 0.01 (dashed), and 0.08 (dotted). (c) The dependence of bulk mode conversion efficiency $\Lambda$ on the collision frequency $v / \omega[13]$.

is added, the dispersion curves are separated because the discriminant in (2) includes the imaginary part and cannot be zero. Since some amount of the helicon wave energy directly decays due to the finite collision, the energy that converts to the TG wave reduces as $v / \omega$ increases. The separation between two dispersion curves also increases as $v / \omega$ does, reducing the efficiency of the bulk mode conversion. In this case, some fraction of the helicon wave energy converts to TG wave energy, and the rest of the helicon wave energy directly dissipates via collision due to its large imaginary part $\left(k_{\perp \text { imag }} x_{0} \sim 1\right.$ at $\left.v / \omega=0.08\right)$ around the MCS. Figure 4 (c) shows the dependence of the bulk mode conversion efficiency $\Lambda=\Gamma_{\mathrm{TG}} / \Gamma_{\text {Helicon }}$ on the collision frequency $v / \omega$, where $\Gamma_{\mathrm{TG}}$ and $\Gamma_{\text {Helicon }}$ are the energy fluxes of the TG and the helicon waves, respectively, when evaluated near the MCS. One may note that the bulk mode conversion efficiency decreases monotonically when $v / \omega$ increases.

\section{Resonance and Anti-Resonance Properties of Helicon Discharge}

The concept of the "resonance" wave discharge was introduced by Shamrai and Taranov to explain the high power absorption efficiency of the helicon plasma source
$[39,40]$. The oscillations of any bounded system driven by an external source can be represented as a superposition of multiple waves. When the driving frequency is close to some of the eigenfrequencies of the system, the amplitude of the specific mode grows sharply. The wave fields are represented as Fourier series in the longitudinal $k_{\|}$and azimuthal $m$ modes. Substituting these wave fields into the Maxwell equations and the cold electron momentum equation give the wave field amplitudes for each component. The field amplitudes in the cylindrical helicon plasma are represented by the superposition of the helicon wave amplitude $A_{\mathrm{H}}$ and the TG wave amplitude $A_{\mathrm{TG}}$ with the basis function of the $m^{\text {th }}$ order Bessel function $J_{m}\left(k_{\perp \pm r}\right)$, where $m$ is the azimuthal mode number and $k_{\perp \pm}$ are the transverse wavenumber of the helicon wave $\left(k_{\perp_{-}}\right)$and the TG wave $\left(k_{\perp+}\right)$, respectively.

In typical laboratory experiments, plasma is surrounded by insulating walls made of quarts or glass. Since the small phase shift introduced by a real glass or quartz tube does not affect the wave field calculation [95], we can treat the insulator as having a dielectric constant of unity (vacuum) for simplicity. The waves in the vacuum region are highly evanescent since the axial wavenumber of the helicon waves $k_{\|}$is typically larger than $k_{0}=\omega / c$ by two orders of magnitude [93]. Therefore, the wave fields in the vacuum are represented by the superposition of the amplitudes of the TM mode $\left(A_{\mathrm{TM}}\right)$ and the TE mode $\left(A_{\mathrm{TE}}\right)$, whose profiles are written using the modified Bessel function $I_{m}\left(k_{\|} r\right)$ and McDonnel function $K_{m}\left(k_{\|} r\right)$. The electromagnetic field excited in the plasma by the antenna can be obtained by connecting the wave field in the plasma and in the vacuum across the plasma surface $\left(r=r_{0}\right)$ where the antenna current is supplied. The conditions to be imposed are as follows: the continuity of the tangential electric field,

$$
E_{\theta, z}\left(r=r_{0}+0\right)=E_{\theta, z}\left(r=r_{0}-0\right)
$$

and the Faraday's law across the plasma surface layer,

$$
\begin{aligned}
& B_{\theta}\left(r=r_{0}+0\right)-B_{\theta}\left(r=r_{0}-0\right)=-i \frac{m}{k_{\|} r} N I, \\
& B_{z}\left(r=r_{0}+0\right)-B_{z}\left(r=r_{0}-0\right)=N I,
\end{aligned}
$$

where $I$ is the antenna current multiplied by some constant. The four unknown wave amplitudes are the helicon $\left(A_{\mathrm{H}}\right)$ and TG $\left(A_{\mathrm{TG}}\right)$ waves in the plasma and the TM $\left(A_{\mathrm{TM}}\right)$ and TE $\left(A_{\mathrm{TE}}\right)$ modes in the vacuum, which are found by using the above four joining conditions. This joining procedure yields the following constants for the helicon and the TG wave amplitudes [41],

$$
A_{\mathrm{H}}=-\beta \frac{D_{+}}{D} I, A_{\mathrm{TG}}=-\beta \frac{D_{-}}{D} I .
$$

$D$ and $D \pm$ are the dispersion functions defined as

$$
\begin{aligned}
& D \equiv-D_{+} D_{\mathrm{TE}}+D_{-} J_{m}\left(k_{\perp \pm} r_{0}\right), \\
& D_{ \pm}=J_{m}^{\prime}\left(k_{\perp \pm} r_{0}\right)+\frac{m}{k_{\|} r_{0}} J_{m}\left(k_{\perp \pm} r_{0}\right),
\end{aligned}
$$


where $r=r_{0}$ is the plasma radius and $D_{\mathrm{TE}}$ is the dispersion function of the TE mode, which is defined as follows, with the outer conducting boundary at $r=R\left(R>r_{0}\right)$ where tangential electric fields $E_{\theta}(r=R)$ and $E_{z}(r=R)$ are vanished,

$$
\begin{aligned}
& D_{\mathrm{TE}}=-\delta D_{-}+J_{m}\left(k_{\perp-} r_{0}\right), \\
& \delta\left(r_{0}, R\right)=-\beta \frac{\Delta_{01}\left(r_{0}, R\right)}{\Delta_{11}\left(r_{0}, R\right)},
\end{aligned}
$$

where

$$
\begin{aligned}
& \Delta_{01}\left(r_{0}, R\right)=I_{m}\left(k_{\|} r_{0}\right)-\frac{K_{m}\left(k_{\|} r_{0}\right) I_{m}^{\prime}\left(k_{\|} r_{0}\right)}{K_{m}^{\prime}\left(k_{\|} R\right)}, \\
& \Delta_{11}\left(r_{0}, R\right)=I_{m}^{\prime}\left(k_{\|} r_{0}\right)-\frac{K_{m}^{\prime}\left(k_{\|} r_{0}\right) I_{m}^{\prime}\left(k_{\|} r_{0}\right)}{K_{m}^{\prime}\left(k_{\|} R\right)} .
\end{aligned}
$$

The dispersion function $D$ in the denominators in (10) determines the resonance properties of the helicon and TG waves. These fields of the appropriate mode grow near $D=0$; such resonance properties are well established for bounded plasmas. However, the functions $D_{ \pm}$in the numerators in (10) determine the anti-resonance properties of these waves, which are suppressed near $D_{ \pm}=0$. In the absence of the dissipation $(v=0)$, when the dispersion functions satisfy $D=0$ or $D_{ \pm}=0$ in appropriate modes, these resonance and anti-resonance properties are displayed in the excited wave fields. However, when a finite dissipation is included $(v \neq 0)$, the dispersion functions of $D$ and $D_{+}$cannot be zero $\left(D \neq 0\right.$ and $\left.D_{+} \neq 0\right)$ due to the large imaginary part of the transverse TG wavenumber $\left(k_{\perp+, \text { imag }} r_{0} \gg 1\right)$, destroying the resonance properties of the helicon and TG waves $(D=0)$ and the antiresonance property of the helicon wave $\left(D_{+}=0\right)$. Since the imaginary part of the helicon wavenumber is very small $\left(k_{\perp+\text {,imag }} r_{0} \ll 1\right)$, only the anti-resonance property of the TG wave $\left(D_{-}=0\right)$ survives even when the dissipation is included. This is in the case of a wide gap $\left(R \gg r_{0}\right)$ between the plasma $\left(r=r_{0}\right)$ and outer conducting boundary $(r=R)$, which is normally satisfied in the experiment. Shamrai and Taranov also showed that helicon resonances can survive in case of the narrow gap $\left(R \sim r_{0}\right)$ [41]. Arnush and Chen [12,93] also investigated these properties using the HELIC code, which can calculate the onedimensional wave and power absorption profiles of the helicon and the TG waves for more realistic experimental conditions, such as non-uniform plasma density, electron temperature, and neutral gas density with an arbitrary antenna type. These resonance or anti-resonance properties result in non-monotonic power absorption dependence on the plasma density and magnetic field strength [41-43].

\section{Mode Jumps in Helicon Discharge}

In numerous helicon experiments, abrupt changes in the discharge regime have been observed as the plasma density jumps. Figure 5 [1] shows an example of the density jump observed by varying the magnetic field. The plasma density does not change linearly with $B_{0}$ (along the

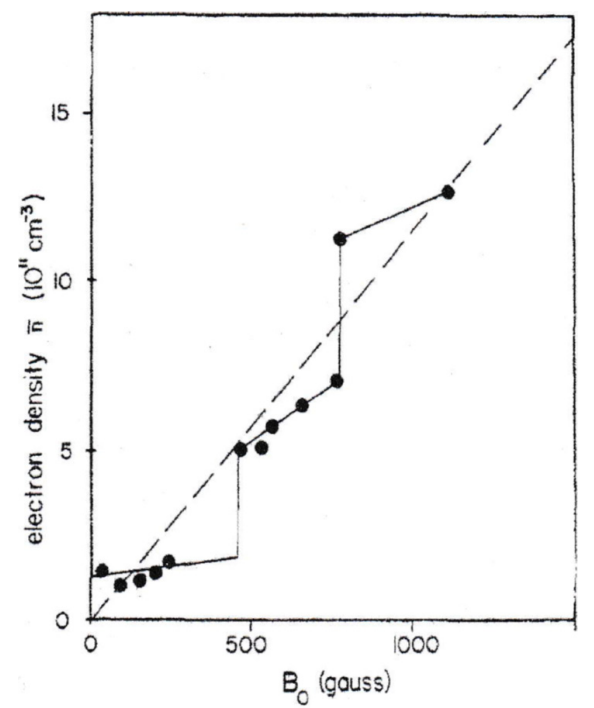

Fig. 5 Variation of the average electron density $\bar{n}$ with $B_{0}$. The solid curve is fitted to the experimental points and the broken curve is the dispersion relation of whistler wave [1].

broken curve for plane wave whistler propagation) as indicated by (7). The first jump occurs at $B_{0} \sim 500 \mathrm{G}$, where the electron density is five times greater than before the jump and the central core of the plasma turns light blue due to strong emission of ArII lines. Further increasing $B_{0}$ causes a second density jump at $B_{0}=750 \mathrm{G}$. Both of these density jumps accompany the variation of the axial wavelength, meaning that the linear relationship (7) with fixed $k_{\|}$is not satisfied.

Chi et al. [59] found the presence of the four jumps by increasing the RF power, as shown in Fig. 6. The first two jumps at low RF power are identified as $\mathrm{E}-\mathrm{H}$ and $\mathrm{H}-\mathrm{W}$ transitions. In this case, three different $\mathrm{W}$ modes (named $\mathrm{W}_{1}, \mathrm{~W}_{2}$, and $\mathrm{W}_{3}$ ) are seen. These three modes correspond to the different helicon wave modes. The wave vectors $\left(k_{\perp}\right.$, $\left.k_{\|}\right)$for these modes are $(0.33,0.21),(0.55,0.21)$ and $(0.80$, 0.28 ) in units of $\mathrm{cm}^{-3}$ for $\mathrm{W} 1, \mathrm{~W} 2$, and $\mathrm{W} 3$, respectively. They measured the amplitude and phase of the wave magnetic fields along the axis for each mode. For the first two modes, it was found that wave fields exist near the antenna, but only small-amplitude axial standing waves were found in the diffusion chamber, indicating that the helicon wave was not effectively excited. Therefore, in these two discharge modes, the near fields of the antenna play a dominant role on plasma production, as expected for the $\mathrm{E}$ and $\mathrm{H}$ modes [47]. In the three $\mathrm{W}$ modes, large-amplitude axial waves were observed. These wave structures showed partial modulation in the wave amplitude due to the interference of incident waves reflected from the end plate of the diffusion chamber, indicating that the three $\mathrm{W}$ modes form a partially traveling and partially standing wave structure. The right panel in Fig. 6 shows the mode transitions of $\mathrm{W} 1-\mathrm{W} 2-\mathrm{W} 3$ in $k$ space. The $\mathrm{W} 1$ and $\mathrm{W} 2$ modes share 

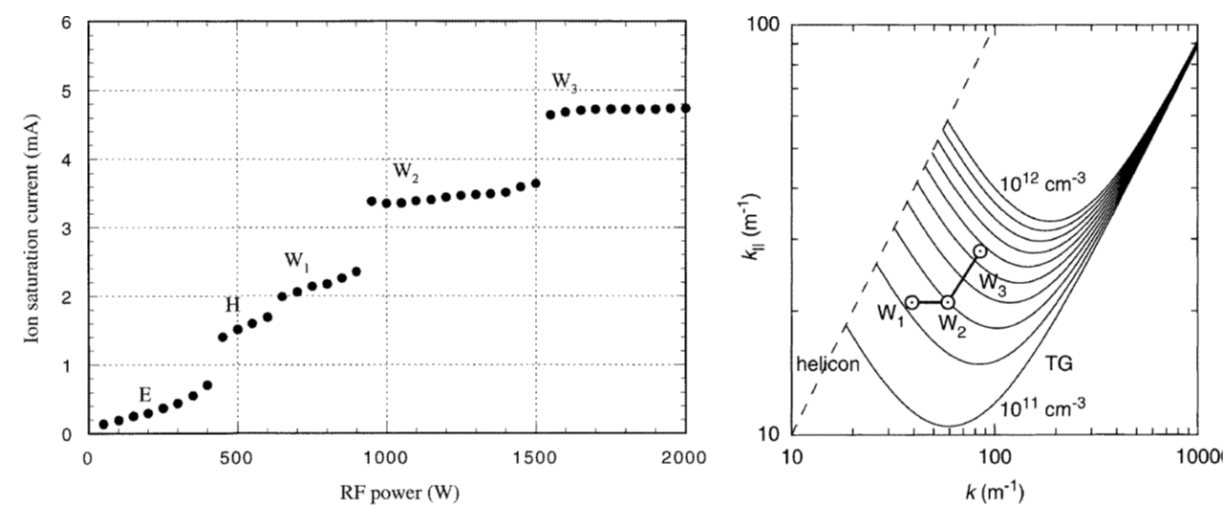

Fig. 6 Left panel: ion saturation current (i.e. plasma density) as a function of RF power. Right panel: transition diagram for the wavemodes in $k$ space. The horizontal and vertical axes are total and parallel wavenumbers, respectively. Dispersion relations (2) are shown for $n=10^{11} \mathrm{~cm}^{-3}$ to $n=10^{12} \mathrm{~cm}^{-3}$ in steps of $n=10^{11} \mathrm{~cm}^{-3}[59]$.

the same axial mode number, but they have different perpendicular wavenumbers at different plasma densities, as expected from the dispersion relation (2). In contrast to the $\mathrm{W} 1$ and the $\mathrm{W} 2$ modes, the $\mathrm{W} 3$ has a higher axial wavenumber. The E-H mode transition exhibits hysteresis $[60,96,97]$, which can qualitatively be understood in terms of an electron power balance $[60,61,98]$ wherein the power absorbed by the plasma has a nonlinear dependence on the electron density. The equilibrium state plasma densities are determined at the intersections of the two curves of the absorbed power and the dissipated power, displayed in the plane of the power and density. When there are multiple solutions for a given value of the coil current due to the nonlinearity of the absorbed power curve, a range of values of the coil current that permit either $\mathrm{E}$ or $\mathrm{H}$ mode discharges exist, resulting in the hysteresis feature of the mode transition [98]. This dependence is also intrinsic for the density jump in the helicon plasma. Indeed, the theory predicts that the nonlinearity of the power absorption curves arises from decreasing the power absorption at the anti-resonance of the TG wave [41-43]. The power balance between the dissipated power and the absorbed power predicts a stable discharge in several density modes and the possibility of abrupt transitions between them.

Figure 7 [43] shows the total power absorption and dissipated power dependences of the plasma density (assuming uniformity) for a Nagoya-type antenna at different currents that were calculated using the following parameters: background magnetic field $B_{0}=250 \mathrm{G}$, RF frequency $\omega_{\mathrm{RF}} / 2 \pi=13.56 \mathrm{MHz}$, collision frequency $v=10^{7}$ $1 / \mathrm{s}$ (Ar pressure approximately $1 \mathrm{mTorr}$ ), plasma radius $r_{0}=7.5 \mathrm{~cm}$, conducting cylinder radius $R=10 \mathrm{~cm}$, and the device length $L=50 \mathrm{~cm}$. The power absorption curves vary non-linearly with the plasma density. The antiresonance of the TG wave occurs when the boundary value of the helicon wave $E_{\theta, \mathrm{H}}$ has a node at the plasma surface $\left(r=r_{0}\right)$, that originates from the zero-point of the Bessel function describing the helicon wave [41]. The boundary value of the $E_{\theta, \mathrm{H}}$ oscillates as density increases since the

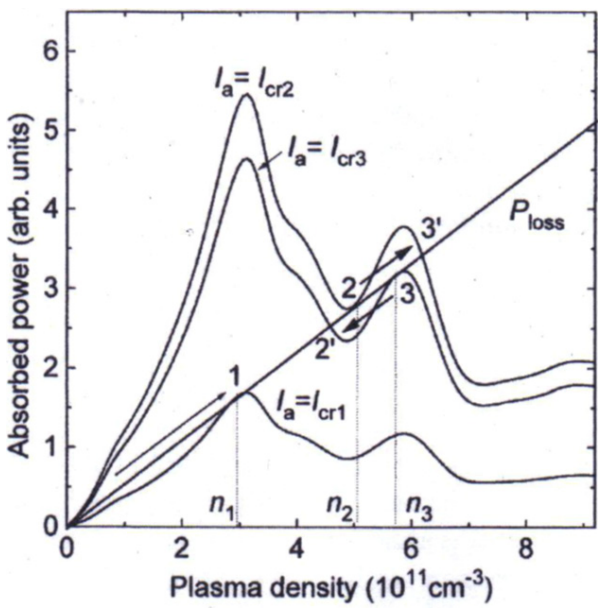

Fig. 7 Absorbed and dissipated power $P_{\text {loss }}$ curves demonstrating direct (2 to 3 ') and back ( 3 to 2') density jumps, which show the origin of hysteresis of density as a function of RF power which corresponds to antenna current $I_{\mathrm{a}}[43]$.

radial scale of the helicon wave decreases, as confirmed by the experiment [99]. The two minimums seen in Fig. 7 are originally related to the first and the second radial modes. Forming a discharge equilibrium is possible at the intersections of the absorbed power and dissipated power, and the stabilities of the equilibrium states are determined by conducting a stability analysis using a small fluctuation [43]. The stability analysis shows that the equilibrium state is stable against fluctuations increasing the plasma density at $n_{1}<n<n_{2}$ and $n_{3}<n$ and unstable at $n_{2}<n<n_{3}$. As seen in Fig. 7, the discharge begins provided that the antenna currents exceed the critical value $I_{\mathrm{cr} 1}$ and the plasma density grows continuously as the antenna current (the RF power) increases from $I_{\mathrm{cr} 1}$ to $I_{\mathrm{cr} 2}$. The dissipation curve touches the absorption curve from below at point 2 , where the equilibrium state is unstable, resulting in a discontinuous jump to a higher density at point 3', which is a stable equilibrium point. If the antenna current decreases, the 
back transition between 3-2' occurs at $I_{\mathrm{cr} 3}<I_{\mathrm{cr} 2}$. Thus, hysteresis is intrinsic to density jumps. Multiple transitions at varying input power accompanying hysteresis are observed in the experiment [99]. A direct experimental and theoretical comparison of the antenna loading and density jumps was carried out by Shinohara and Shamrai [100], and showed satisfactorily agreements within $50 \%$ of the threshold density (just after the density jump) over a wide range of operation parameters.

Calculations by Chen and Torreblanca [101] using the HELIC code predict a sudden density jump at a threshold RF power depending on the ratio of plasma loading resistance to circuit resistance. Figure 8 [101] shows equilibrium densities with varying input power $P_{\mathrm{RF}}$ for the three values of the circuit resistance $R_{\mathrm{c}}$. As seen in Fig. 8, when measured experimentally, the threshold power $(\sim 40 \mathrm{~W})$ and plasma density ( $\sim 10^{12} \mathrm{~cm}^{-3}$ after the jump) show the good agreement with the prediction in the case of $R_{\mathrm{c}}=1 \Omega$. This is in rough agreement with the experimentally observed value of $R_{\mathrm{c}} \sim 0.7 \Omega$ in the measured density jump. It was also suggested that the observed density jump is not from an ICP mode into a helicon mode since the finite magnetic field $\left(B_{0}=80 \mathrm{G}\right)$ precludes the ICP mode in the magnetized system and may be a low-density helicon mode. In the magnetized ICP (MICP) modes, there are two regions called skin $\left(\omega_{\mathrm{RF}}>\omega_{\mathrm{ce}}\right)$ and wave effect dominant $\left(\omega_{\mathrm{RF}}<\omega_{\mathrm{ce}}\right)$ regions [102]. The skin effect dominant region is similar to unmagnetized plasma (ICP). In this region, the waves decay inside the skin depth region and electromagnetic oscillations exist at skin depth only. In the wave effect dominant region, there is a wave penetration into the plasma (helicon plasma) [102-106].

In this experiment, the $\mathrm{H}-\mathrm{W}$ mode transition is understood to be the transition from the axial standing wave structure (the wavenumber of which is nearly the same as the fundamental excited antenna wavenumber) to the axial traveling wave structure (the wavenumber of which satisfies the helicon's dispersion relation) [57]. Although this seems to be a reasonable way to distinguish the $\mathrm{H}$ mode

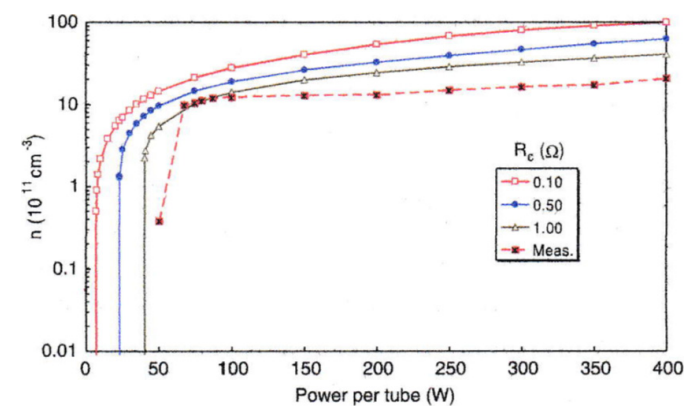

Fig. 8 Density versus $P_{\mathrm{RF}}$ for three values of circuit resistance $R_{\mathrm{c}}$, showing the abrupt jump in density as applied power is increased. The dashed curve shows density measured inside the discharge tube [101]. from the $\mathrm{W}$ mode, uncertainty still remains, as Chen and Torreblanca pointed out [101]. In a low-density region, the helicon wave cannot propagate as shown in Fig. 1; in this region, it becomes an evanescent wave (penetrates within the skin depth $\delta=2 \pi / k_{\mathrm{H} \text {, imag }}$ ) with wave structures (standing wave structures) similar to those of ICP fields. However, as seen in Fig. 1, the TG wave can propagate and contribute to the power deposition in low-density plasma. From (2), we can obtain the approximated TG wavenumber (real part $k_{\perp, \mathrm{TG}}$ and imaginary part $k_{\mathrm{TG} \text {, imag }}$ ) at $\alpha \ll 1$ (that is $n \ll n_{\text {up }}$ ) as follows [42],

$$
k_{\perp, \mathrm{TG}}=k_{\|} \frac{\omega_{\mathrm{ce}}}{|\gamma|^{2} \omega_{\mathrm{RF}}}, k_{\mathrm{TG}, \mathrm{imag}}=-k_{\|} \frac{v}{\omega_{\mathrm{RF}}} \frac{\omega_{\mathrm{ce}}}{|\gamma|^{2} \omega_{\mathrm{RF}}} .
$$

With fixed $k_{\|}$, when the magnetic field is sufficiently strong $\omega_{\mathrm{RF}} \ll \omega_{\text {ce }}$, since the TG wave is highly electrostatic $k_{\perp} \gg k_{\|}$and highly dissipative $k_{\mathrm{TG}}$, imag $r_{0} \gg 1$, this wave structure cannot be observed and has no effect on the observed wave field structures of the magnetic wave field components. On the other hand, when the magnetic field is small, the TG wave can be observed. Indeed, the low-density mode sustained by the TG wave is observed by Lho et al. [92]. Figure 9 shows the comparison of the wave dispersion related to the experimental data. The observed wave fields at the first harmonic (drive frequency is $f=13.56 \mathrm{MHz}$ ) are identified as TG waves (pseudosurface waves) at a low-density $\left(n \sim 9.0 \times 10^{10} \mathrm{~cm}^{-3}\right)$ and high $B$ field $\left(\omega_{\text {ce }} / \omega>2.5 \sim B_{0}=12 \mathrm{G}\right)$, while waves at the second harmonic (drive frequency is $f=27.12 \mathrm{MHz}$ ) behave as helicon waves at a low $B$ field $\left(\omega_{\text {ce }} / \omega<2.5 \sim\right.$ $\left.B_{0}=24 \mathrm{G}\right)$. By using high-frequency $f=50 \mathrm{MHz}$ and

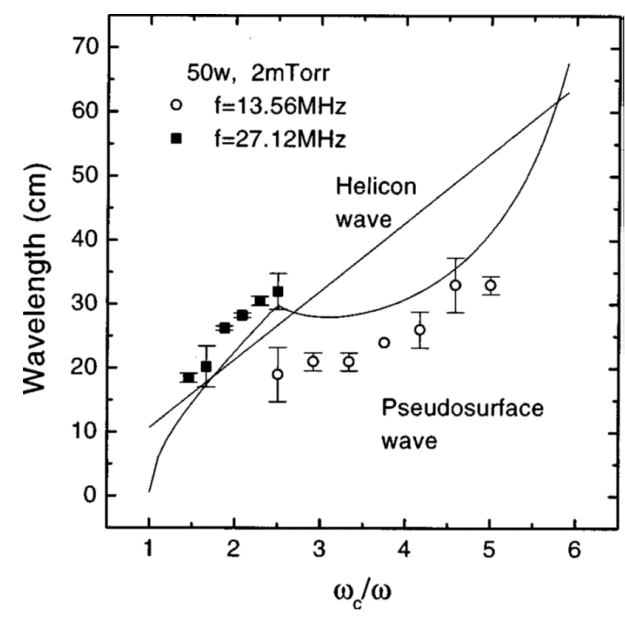

Fig. 9 Comparison of the wave dispersion relation to experimental data. Waves of the first harmonic ( $f=$ 13.56 MHz) follow the pseudosurface wave dispersion relation, while waves of the second harmonic ( $f=$ $27.12 \mathrm{MHz}$ ) follow the helicon wave. The deviation of experimental data from the helicon dispersion relation might come from the uncertainty of the density measurement [92]. 
very-high-frequency $f=144 \mathrm{MHz}$, Sakawa et al. [107] also observed low-density $\left(\sim 10^{10} \mathrm{~cm}^{-3}\right)$ discharge being sustained by the TG wave.

\section{Density Limit and Neutral Deple- tion}

A higher-density helicon source is useful for applications such as the plasma process, the nuclear fusion, and the electric thruster. If a peak density of order $10^{21} \mathrm{~m}^{-3}$ can be achieved, such plasma would be useful for accelerator applications [108]. One of the advantages of a helicon source is its wide variation of device scales. Shinohara and Tanikawa $[4,109,110]$ demonstrated excellent particle production efficiency $N_{\mathrm{e}} / P_{\text {inp }}\left(N_{\mathrm{e}}\right.$ is the total number of electrons in a whole plasma and $P_{\text {inp }}$ is the input RF power) at various device scales from small (plasma volume of $23 \mathrm{~cm}^{3}$ ) to large (plasma volume of $2.1 \mathrm{~m}^{3}$ ). Figure 10 [110] shows the relationship between $N_{\mathrm{e}} / P_{\text {inp }}$ and the device cross section $a^{2}$ ( $a$ is the device radius). As seen in Fig. $10, N_{\mathrm{e}} / P_{\text {inp }}$ is proportional to $a^{2}$, as expected from the classical diffusion (in this case, primarily radial diffusion [109]).

Maximum peak densities of order $10^{20} \mathrm{~m}^{-3}$ are reported in some experiments [35,44]. Miljak and Chen [24] have tried to produce higher-density helicon plasma with two helical antennas to excite $m=+1$ waves, using $2 \mathrm{~kW}$ of RF power on each antenna. They observed a maximum plasma density of $8.0 \times 10^{19} \mathrm{~m}^{-3}$ and found that higher density could not be achieved by increasing the pressure and moving the antennas. They suggest the density limit results from neutral depletion.

There are two regimes of collisional and collisionless plasma in which neutral depletion occurs in different ways [23]. In collisional plasma, neutral depletion occurs due to the pressure balance between plasma and neutrals, which plasma and neutrals exert on each other due to mutual ion-neutral collisions. This process of neutral depletion is called neutral-pumping [111-114]. In collisional

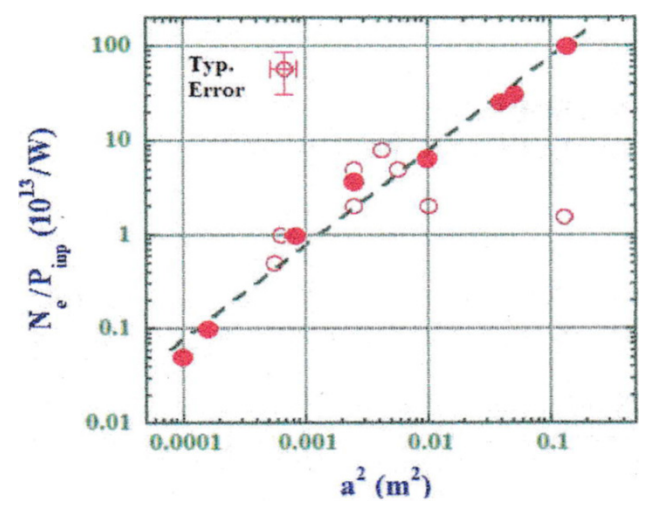

Fig. 10 Relationship between particle production efficiency $\left(N_{\mathrm{e}} / P_{\text {inp }}\right)$ vs. device cross section $\left(a^{2}\right)$ for different helicon sources [110]. plasma, neutral depletion is more pronounced with neutral gas heating since the total pressure balance changes due to the neutral density being depleted in the high neutral temperature region [103, 115-119]. In collisionless plasma, neutral depletion occurs only due to electron impact ionization followed by rapid movement from the plasma to the chamber wall via the presheath electric field. This process of neutral depletion is called ion-pumping [112, 120,121]. In RF plasma sources, the effects of the neutral depletion have been observed in electron cyclotron resonance (ECR) plasma [103, 115, 122], ICP [117-121, 123, 124], and helicon plasma [25,86, 120, 125-131].

Gilland et al. [125] observed the effect of neutral depletion in helicon plasma, and showed that the neutral pressure in the bulk of a helicon discharge decreases by a factor of ten compared with the pressure before the discharge. Aanesland et al. [127] directly measured the neutral gas density by spatially resolved laser-induced fluorescence spectroscopy with two-photon excitation (TALIF). Figure 11 (a) shows the neutral depletion $D=n_{\mathrm{Xe}}$ on $/ n_{\mathrm{Xe}}$, off, where $n_{\mathrm{Xe}}$, on and $n_{\mathrm{Xe}}$, off are the relative neutral densities when the plasma is on and off as a function of the radius for three different magnetic fields $(0,95$, and $150 \mathrm{G})$. The largest neutral depletion is obtained in the center of the discharge for all these cases and increases with an increasing magnetic field. Figure 11 (b) shows the plasma pressure ( $P_{\mathrm{e}}=n_{\mathrm{e}} k_{\mathrm{b}} T_{\mathrm{e}}$, where $k_{\mathrm{b}}$ is the Boltzmann constant) and the neutral pressure $\left(P_{\mathrm{g}}=n_{\mathrm{g}} k_{\mathrm{b}} T_{\mathrm{g}}=P_{\text {fill }}\right.$, where $P_{\text {fill }}$ is the fill pressure before discharge, $n_{\mathrm{g}}$ is the neutral density, and
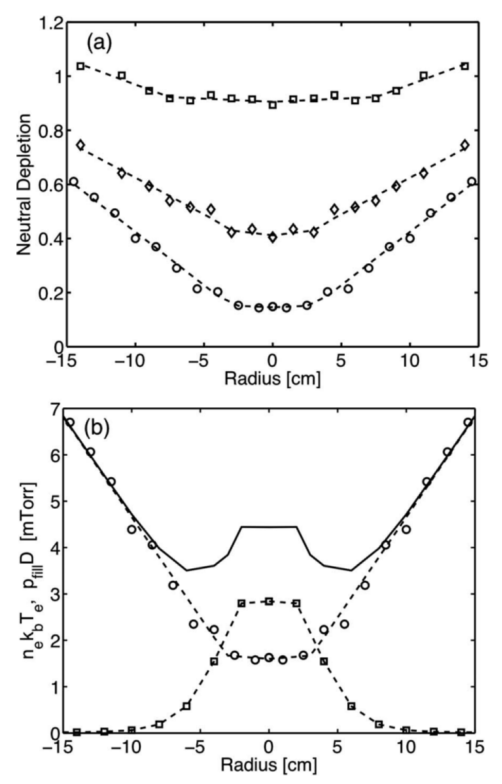

Fig. 11 (a) Neutral depletion as a function of radius, where squares, diamonds, and circles correspond to 0,95 , and $150[\mathrm{G}]$, respectively. (b) Plasma pressure (squares) and neutral pressure (circles) as a function of radius, the solid line being the sum of the two [127]. 
the neutral gas temperature $T_{\mathrm{g}}$ is assumed to be constant). Both $P_{\mathrm{e}}$ and $P_{\mathrm{g}}$ are nonuniform in the radial direction with gradients pointing inward and outward, respectively. The ions and neutrals are pushed by these gradients, and mutual collisions with each other yield an additional drag force acting in the opposite direction. Therefore, the drag force on the ions due to collisions with neutrals points inward, while the drag force on the neutrals by collisions with ions points outward, which results in a neutral depletion at the center of the discharge. The unbalance of the total pressure in Fig. 11 (b) may result from ignoring the effects of the neutral heating and magnetic pressure [127].

Clarenbach et al. [128] measured the time-dependence of the temperature and the density of the Ar* $\left({ }^{3} P_{0}\right)$ metastable atoms. From the time variation of the Ar* $\left({ }^{3} P_{0}\right)$ density, the density of the ground-state argon atoms can be deduced. They showed that, when the discharge changed from ICP to helicon discharge, argon atom depletion of up to $90 \%$ and ionization degrees of up to $65 \%$ were achieved in the antenna zone. Magee et al. [25] also measured the time-resolved neutral density at and downstream from the RF antenna and observed slower neutral depletion ( $\sim 14 \mathrm{~ms}$ ) compared with the decreasing timescale of the neutral density underneath the antenna $(\sim 15 \mu \mathrm{s}$, which may be due to the high ionization rate). Magee et al. [129] also showed a hollow neutral density profile such that the core density was 20th of the edge density, which may be due to the ion-pumping effect rather than neutral-pumping. By using the collisional radiative (CR) model [130], Denning et al. [131] observed evidence of enhanced axial plasma velocity as a consequence of reduced drag by the neutrals due to depletion.

Fruchtman et al. [111] analytically revealed the effect of neutral depletion on unmagnetized plasmas in pressure balance with neutral gas by self-consistently solving the plasma and neutral dynamics. Figure 12 shows the plasma and neutral density profile calculated for two cases; the first case is a discharge in which the total neutral number $N_{\mathrm{T}}$ is fixed, and in the second case the neutral density at the plasma boundary $N_{\mathrm{W}}$ is fixed by using the following parameters: plasma size $a=5 \mathrm{~cm}(\xi=x / a)$, the neutral pressure prior to the discharge $N_{\mathrm{W}} T_{\mathrm{g}}=10 \mathrm{~m}$ Torr (assuming $T_{\mathrm{g}}$ is fixed as the room temperature), and two different power levels (low and high). When the input power is low, the neutral density almost does not vary; however, when the power is high, in both cases, the plasma density sharply drops to zero and the neutral density sharply peaks to a high density near the boundary. These results are qualitatively consistent with those of the experiment [121]. They also showed that the neutral depletion enhances plasma transport, which can result in an unexpected decrease of the plasma density when the power is increased despite the increase of plasma production with power. In the collisional regime, where the pressure balance is maintained, Liard et al. [132, 133] and Fruchtman [134] included the effect of the neutral gas heating. They showed that neu-
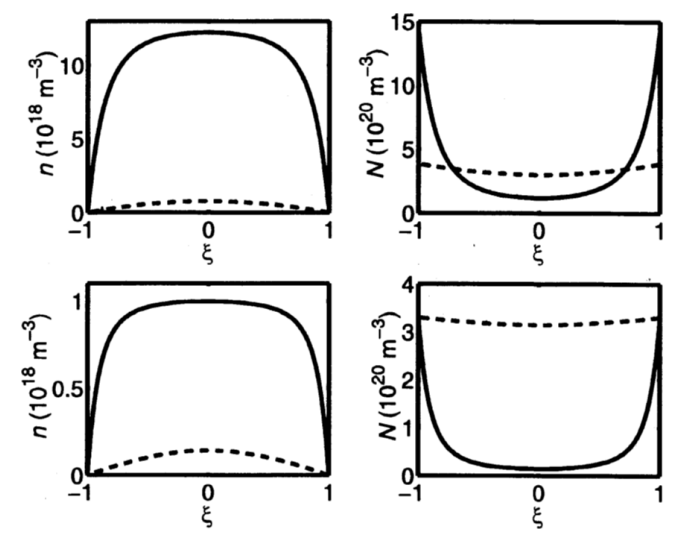

Fig. 12 Plasma and neutral density profiles. Top panel: fixed total neutral number with $P=33$ (dotted line) and 509 (solid line) $\mathrm{kW} / \mathrm{m}^{2}$. Bottom panel: fixed neutral density at the boundary with $P=3$ (dotted line) and 121 (solid line) $\mathrm{kW} / \mathrm{m}^{2}[111]$.

tral depletion and plasma transport are enhanced due to gas heating.

Raimbault et al.[114] extended the steady-state isothermal model with three neutral pressure regimes according to the three well-established theories: the lowpressure regime (the product of the pressure with the discharge length $p L<0.1 \mathrm{mTorr} \cdot \mathrm{m})$ with the Tonks and Langmuir model[135], the high-pressure regime ( $p L>10 \mathrm{mTorr} \cdot \mathrm{m})$ with the Schottky model [136], and the intermediate-pressure regime with the Godyak model [137], which bridges the gap between the low- and highpressure regimes. They predict that the neutral density can present a maximum at the center of the discharge when the drag on neutrals due to collisions with ions is negligible (the Tonks and Langmuir model). In this case, the neutral dynamics is governed by a balance between the inertial and pressure terms of neutrals rather than the total pressure balance between plasma and neutrals [114]. Fruchtman et al. $[112,113,138]$ call this centrally peaked neutral density profile neutral repletion and predicts that neutral repletion will occur when the ionization is intense and the neutrals are sufficiently collisionless.

The effect of the magnetic field on neutral depletion was studied by Liard et al. [139] and Fruchtman [140,141]. Fruchtman [141] points out that the rate of neutral-gas depletion is determined by the plasma outward flux at the boundaries and that the neutral-gas depletion increases when this flux increases. When the plasma density is kept constant while the magnetic field is varied, the plasma particle flux decreases as the magnetic field increases, then neutral depletion decreases with the magnetic field. When the plasma particle flux is kept constant while the magnetic field is varied, neutral depletion does not vary with the magnetic field. In these cases, the magnetic field is assumed to be uniform and the effect of the diamagnetic effect is not included. 
Diamagnetism is a fundamental characteristic of highbeta plasmas. Low-temperature weakly magnetized plasmas can have a high beta value, especially in plasma sources such as helicon plasma [142]. Indeed, the diamagnetic effect in helicon plasma has been observed by Roberson et al. [143] and Takahashi et al. [144-146]. However, some researchers [147-150] report that the magnetic reduction was much smaller than expected with the plasma beta. Scime et al. [147] claim that the diamagnetic effect was small because the MHD equilibrium was not established in his experiment. The cases of a significant diamagnetic effect with neutral depletion have been studied by Fruchtman and Shinohara theoretically [142] and experimentally [151]. They concluded that diamagnetism is weakened if the neutral's pressure is comparable to the plasma pressure and if the coupling of plasma and neutral's pressures by ion-neutral collisions is sufficiently strong. Figure 13 [151] shows the dependence of the magnetic field reduction rate $R=\left|\Delta B / B_{0}\right|$ on $B_{0}$ at the neutral pressure $P_{0}=0.30 \mathrm{~Pa}$. By considering the pressure balance between the plasma and the magnetic field, the magnetic field reduction rate can be written as $R_{\mathrm{I}}=1-\sqrt{1-\beta}$. When the neutral pressure is included in the total pressure balance, the magnetic field reduction rate can be written as $R_{\mathrm{II}}=1-\sqrt{1-\beta-\Delta \beta_{n}}$, where $\Delta \beta_{n}=\left(P_{g}-P_{g 0}\right) /\left(B_{0}^{2} / 2 \mu_{0}\right)=\beta_{n}-\beta_{n 0}\left(\beta_{n 0}\right.$ and $\beta_{n}$ are neutral's beta at the wall and the center of the discharge, respectively). The $R_{\mathrm{I}}$ fitting curve is represented as a black dotted-circle line, and the $R_{\mathrm{II}}$ fitting is represented as "Cal." (with standard ion-neutral collision frequency) and "Cal. (3.5)" (with a collision frequency enhanced by a factor of 3.5) noted lines. The "C" and " $C(3.5)$ " curves indicate whether diamagnetism is expected to be suppressed by neutral depletion (the diamagnetic effect is noticeable when $C \gg 1$ and the diamagnetic effect is small, while the neutral's pressure gradient due to neutral depletion is large when $C \ll 1$ ). As seen from Fig. 13, despite certain simplifying assumptions of MHD equilibrium, there

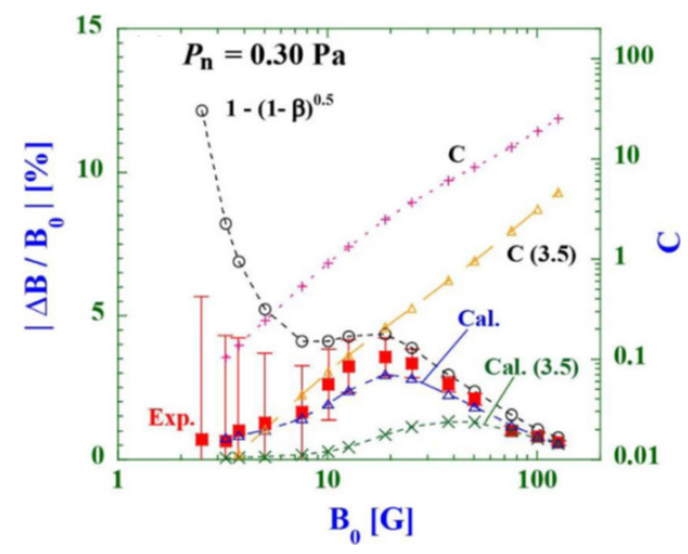

Fig. 13 Measurements of the diamagnetic effect in a helicon source [151]. is good agreement between the experiments and calculated $R_{\mathrm{II}}$ lines. Note that a theoretical $R_{\mathrm{I}}$, neglecting a neutral's pressure effect, can describe the experimentally obtained diamagnetic effect only in the higher $B_{0}$ where $C<1$ is satisfied.

The effects of the neutral depletion on the plasma transport and density limit have been accepted by numerous authors. However, since the precise mechanism of neutral depletion remains unclear, further investigations are needed to overcome this issue to develop higher-efficiency plasma sources.

\section{Diffusion Processes in ICP and He- licon Discharges}

The plasma diffusion process in RF plasma still remains a controversial issue, since the observed plasma density is usually flat or peaked on the axis even when the skin depth of the RF power deposition is much smaller than the plasma radius [152].

In the ICP (unmagnetized) discharge, as seen from Fig. 14 [153], although the RF field from the antenna current penetrates the skin slightly and the electron temperature peaks in the skin layer, the observed profile shows the minimum in the skin layer and peaks near the center. A mechanism to explain this centrally peaked density profile in the ICP is the anomalous skin effect [154-160], which theoretically proposes that electron thermal motions could transport RF energy past the skin layer when the local Ohm's law is violated. Godyak et al. [161-163] have discovered the nonlinear effect of the ponderomotive force and the second harmonic force caused by the nonlinear Lorentz force $F_{\mathrm{L}}=e\left(v_{\mathrm{d}} \times B\right)$ and the convective part of the integral force $F_{\mathrm{I}}=m v_{\mathrm{d}} \nabla v_{\mathrm{d}}$, where $v_{\mathrm{d}}$ is the RF drift velocity and $B$ is the RF magnetic field. Smolyakov et al. [164] showed that the curvature of the magnetic field in planar ICP and nonlinear electron inertia are direct sources of nonlinear poloidal currents $\left(J_{r}\right.$ and $\left.J_{z}\right)$ and the azimuthal magnetic field $B_{\theta}$. Godyak and Kolobov [165] observed

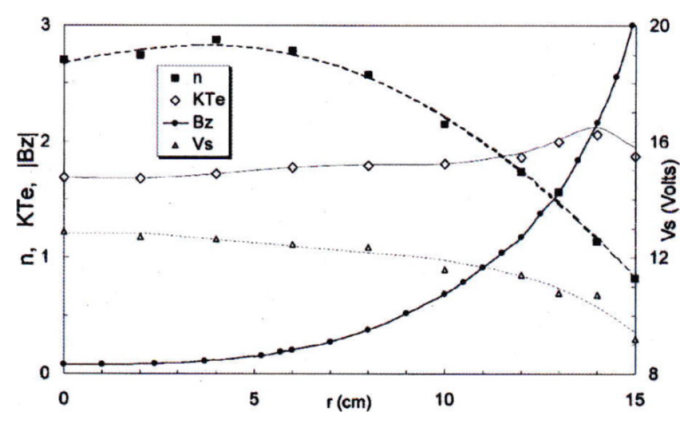

Fig. 14 Spatial profiles of plasma density $n\left(10^{11} \mathrm{~cm}^{-3}\right)$, electron temperature $k_{\mathrm{b}} T_{\mathrm{e}}$, space potential $V_{\mathrm{s}}$, and axial RF magnetic field $B_{z}$ (arbitrary units) in an ICP discharge in $10 \mathrm{mTorr}$ of argon, with $P_{\mathrm{RF}}=300 \mathrm{~W}$ and $f_{\mathrm{RF}}=2 \mathrm{MHz}$ [153]. 
the negative power absorption of the electromagnetic field in a low-pressure ICP discharge. When the electron mean free path is approximately the size of the discharge, the RF current transferred by thermal electron motion can be the opposite in phase to the local electric field, resulting in local negative energy transfer from the RF field to electrons. A simple analytic model describing the negative power absorption was developed by Tyshetskiy et al. [166], who showed the qualitative agreement of the power absorption profile with the experiment. By tracing the electrons' orbit over many RF cycles, Chen and Evans [153,167] showed that the anomalous skin effect is enhanced by the nonlinear Lorentz force and indeed the density is peaked on the axis.

In the helicon (magnetized) discharge, most of the RF power is absorbed by the excitation of the TG mode in a thin layer of the edge boundary. The plasma diffusion across a magnetic field is a long-standing problem and has been studied extensively for decades [168]. The classical ambipolar diffusion across the magnetic field, of which diffusion coefficient $D_{\perp}$ is inversely proportional to the square of the magnetic field $D_{\perp} \propto B^{-2}$ [169], is several orders smaller than the "Bohm diffusion" scaling as $D_{\perp} \propto B^{-1}$ [22]. When the diffusion rate was investigated experimentally [22], the observed value $D_{\perp}=3 \times 10^{3} \mathrm{~cm}^{2} / \mathrm{sec}$ was much higher than the expected classical ambipolar diffusion $D_{\perp}=20 \mathrm{~cm}^{2} / \mathrm{sec}$. As a result of this large discrepancy, Bohm [22] postulated that the fluctuation of the charge density may produce electric fields that give rise to anomalous diffusion across the magnetic field.

The "short-circuit effect" mechanism is suggested by Simon [170, 171], who claims that the ions can diffuse freely across the magnetic field without the restriction of ambipolarity, while electrons do not follow the ions across the magnetic field, but instead travel along the field lines and conducting wall, and eventually maintain the charge neutrality. In his results, the diffusion coefficient shows $B(H)^{-2}$ dependence. Figure 15 [171] shows the observed dependence of the e-folding distance $r_{0}$, which is determined as the solution of the diffusion equation with the diffusion coefficient. Since $r_{0}$ is proportional to the square root of diffusion coefficient $D\left(r_{0} \propto \sqrt{D}\right)$, the data clearly indicate $D \propto B(H)^{-2}$ dependence. He showed that the ions' intrinsic diffusion rate can explain the discrepancy between theory and the experiment without additional mechanisms, such as the anomalous diffusions in Bohm diffusion. The scaling of particle production efficiency $N_{\mathrm{e}} / P_{\text {inp }}$ exhibited in Fig. 10 [110], which is proportional to $a^{2}$, is derived from the diffusion equation assuming the ions' intrinsic diffusion rate. Fruchtman [140] extended the fluid theory with ambipolar and non-ambipolar (short-circuit effect) diffusion limits and showed the differences of the steady-state density profiles with various collision limits. Chen and Curreli $[152,168]$ point out that the short-circuit effect arises from the sheath adjustment at the end plates, which allows electrons to effectively move

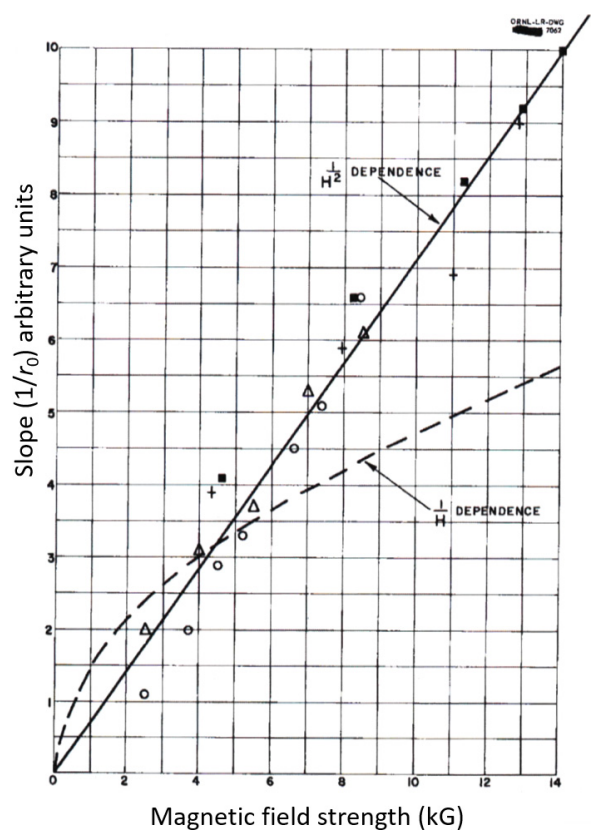

Fig. 15 Plot of slopes $1 / r_{0}$ ( $r_{0}$ is reciprocal e-folding distance of plasma density profile) as a function of magnetic field strength. The e-folding distance $r_{0}$ is proportional to the squared root of diffusion coefficient $\left(r_{0} \propto \sqrt{D}\right)$. $D \propto B(H)^{-2}$ dependence is plotted as straight line (solid line), and $D \propto B(H)^{-1}$ is plotted as curve line (broken line) [171].

across the magnetic field. Lafleur and Boswell [172] investigated cross-field diffusion in low-pressure magnetized plasmas both in the presence and absence of conducting axial boundaries thorough two-dimensional PIC simulation. They found that cross-field diffusion was ambipolar without the axial boundary, but when the axial boundary was added, the diffusion became non-ambipolar in which ions and electrons escaping to the transverse and axial wall, respectively, and total fluxes integrated over all the boundaries were equal.

By using a very long discharge tube in which the short-circuit effect can be avoided, Hoh and Lehnert [173] and Allen et al. [174] showed that the agreement between the experiment and classical diffusion theory was valid only up to a certain critical magnetic field $\left(B_{\mathrm{c}}\right)$. Above the critical magnetic field, an oscillating helical structure appeared, and higher diffusion was present. Bonnal et al. [175] also observed the enhanced transversal flux of ions to the magnetic field above the critical magnetic field. Kadomtsev and Nedospasov [176] investigated the stability problem for the harmonic perturbations in a diffusion model including magnetized electrons. They found the theoretical critical value of the magnetic field (i.e., the onset of the anomalous diffusion) and oscillation frequency of the unstable mode above the critical magnetic field. The relation of the electric and magnetic fields in this oscillation showed the good agreement both qualitatively 
and quantitatively with the measurements performed by Hoh and Lehnert [173]. On the other hand, other experiments [177, 178] report diffusion with the classical rate $\left(D_{\perp} \propto B^{-2}\right)$ even in short cylindrical discharge and strong magnetic fields.

In recent years, Bohm diffusion is predominantly interpreted as an upper limiting value of cross-field diffusion with low-frequency drift-wave fluctuation [179], which plays a dominant role in enhancing classical diffusion $[73,180]$. Density and temperature gradients in magnetized plasma give rise to electron and ion diamagnetic currents across the magnetic field, and the drift velocities associated with these currents excite collective oscillations called drift waves. The effect of drift-wave transport on the plasma confinement has been extensively studied in the magnetic fusion energy research field over past decades [180]. The collisional (resistive) drift-wave instability was discovered and identified by Hendel et al. [73] in a long cylindrical device called the Q machine [181]. A variety of drift-type instabilities, such as the trapped electron modes [182], the trapped ion instability [183], the collisionless curvaturedriven trapped particle mode [184], and the ion temperature gradient-driven drift mode [185], have been observed in the Columbia Linear Machine.

In this chapter, we overviewed the non-classical behaviors of the anomalous skin effect in ICP discharge and the anomalous diffusion across the magnetic field in helicon discharge. Despite the extensive studies on these physical issues, there is currently no consensus on which theory to use to describe diffusion transport.

\section{Instabilities in Helicon Discharge}

Enhanced plasma transport affects the equilibrium plasma behavior [18]. As seen from Fig. 2 and (7), the plasma density is expected to increase monotonically with the magnetic field. Sakawa et al. [66] report that the plasma density does not increase monotonically, but decreases with the magnetic field in light gases. The bottom panel of Fig. 16 [18] shows the measured ion density for four gases, from which one can observe that the light ion [hydrogen $(\mathrm{H})$ ] peaks at a low magnetic field and shows a dramatic decrease of plasma density above the critical magnetic field $\left(B_{\text {crit }}\right)$, while the heavier gases [helium $(\mathrm{He})$, neon (Ne) and argon (Ar)] tend to saturate. Light et al. $[18,82]$ explain these saturations and unexpected decrease of density in the framework of low-frequency electrostatic instability. The upper panel of Fig. 16 shows a spectrogram of the density perturbation $n_{0}\left(B_{0}\right)$ in Ne along with $B_{0}$. One may note that the saturation of $n_{0}$ in the plasma density and onset of strong, low-frequency fluctuations in $n_{0}$ occur at the same $B_{\text {crit }}$. They suggest that the measured density $\left|\nabla n_{0}\right|$ and the space potential profiles $\left|\nabla V_{\mathrm{s}}\right|$ can drive the resistive drift and Kelvin-Helmholtz instabilities, respectively at these low-frequency ranges, and these instabilities may enhance the plasma losses. They also showed
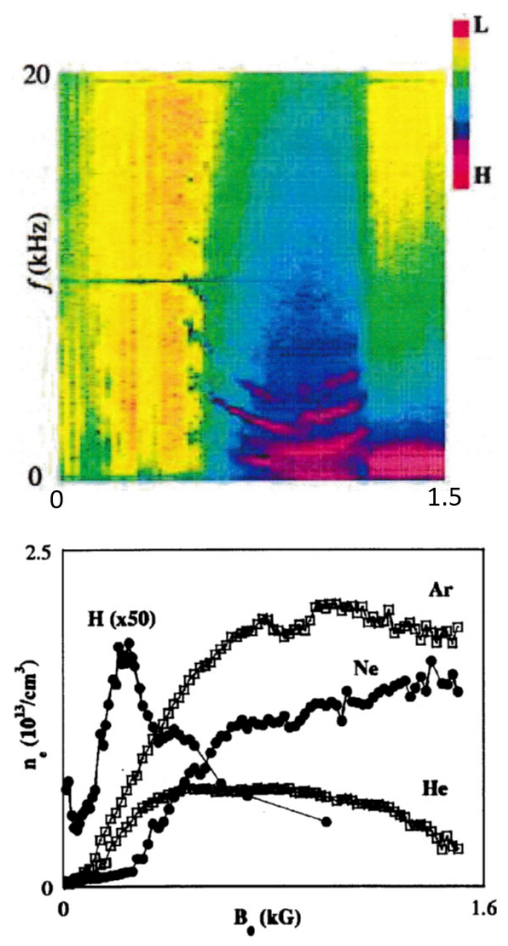

Fig. 16 Equilibrium plasma density vs. magnetic field strength (bottom panel) and spectrogram of the density perturbation $n_{0}\left(B_{0}\right)$ in neon $(\mathrm{Ne})$ along with $B_{0}$ (upper panel) in a helicon source, with $8 \mathrm{mTorr}$ fill pressure and $2 \mathrm{~kW}$ input power [18].

the theoretical growth rates for different ion masses, which showed excellent correlations with the observed equilibrium density decrease, maximum of the measured fluctuation amplitude as a function of magnetic field strength, and onset of maximum fluctuation amplitude for low frequencies in spectrograms.

Tynan et al. $[19,186]$ have observed a radially sheared $E \times B$ azimuthal flow driven by the resistive drift-wave turbulence [187] in the controlled shear decorrelation experiment (CSDX) device. Figure 17 (a) [19] shows the measured time-averaged plasma potential (open circle) normalized to the electron temperature $T_{\mathrm{e}}$ at $r=3 \mathrm{~cm}$, which is the location of the maximum density gradient. The plasma potential peaks at the center of the plasma, has a minimum at approximately $r=4 \mathrm{~cm}$, and then begins to increase in value for $r>4 \mathrm{~cm}$. Thus, a positive radial electric field exists in the inner portion and a negative electric field exists outside of a shear layer $(r \sim 4 \mathrm{~cm})$. The shape of the time-averaged plasma potential profile associated with a radially sheared azimuthal $E \times B$ flow formed via an inverse energy transfer process was predicted [see solid line in Fig. 17 (a)] using theory and numerical simulation [188] and shows excellent agreement with the observation. Figure 17 (b) shows the radial profiles of the low-frequency (i.e., $\omega<\omega_{\text {ci }}$ ) broadband (from $3-4 \mathrm{kHz}$ up to $25-30 \mathrm{kHz}$ ) electrostatic potential fluctuation ampli- 
(a)

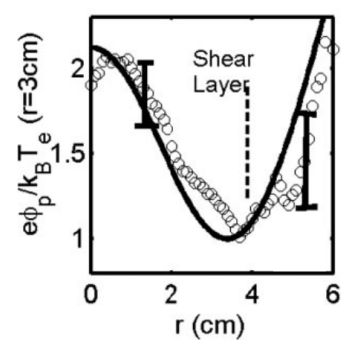

(b)

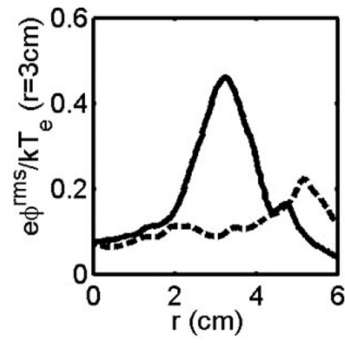

Fig. 17 Time-averaged radial profiles of (a) measured (open circles) and predicted (solid line) plasma potential and (b) broadband potential fluctuation amplitude (solid line) and low-frequency azimuthally symmetric potential fluctuation amplitude (dash-dot line) [19].

tude (solid line) normalized to the electron temperature at $r=3 \mathrm{~cm}$. The electrostatic potential fluctuation has a peak amplitude near the maximum equilibrium pressure gradient at $r=3 \mathrm{~cm}$, which is consistent with expectations for highly resistive drift-wave turbulence [187]. The potential fluctuation also contains a significant component over a frequency band of $0-2 \mathrm{kHz}$ (dash-dot line), which is seen clearly in the $r>4 \mathrm{~cm}$ region, suggesting that there is a radial structure of azimuthally symmetric potential fluctuation. Manz et al. [189] investigated the scenario of transition from an unstable mode to drift-wave turbulence. Antar et al. [190] used a fast-imaging camera to investigate the spatio-temporal properties of radially convective events in the CSDX device. They found that the change of fluctuation structures inside the main plasma column derived from the excitation of different poloidal mode numbers as a function of time.

In the linear Versatile Instrument for studies on Nonlinearity, Electromagnetism, Turbulence, and Applications (VINETA) [191], Grulke et al. [20, 192-196] identified the resistive drift-wave instability by comparing the drift mode density fluctuation characteristics with a linear model proposed by Ellis et al. [75], which reproduced the experimentally observed bent eigenmode structures due to the radial gradients of the collisionality (electron-ion collision frequency) profile. Figure 18 [20] compares the azimuthal mode structure of an $m=5$ drift mode as obtained from the linear dispersion relation calculation with the experimental measurement. A similar mode pattern is obtained from the linear model for experimental radial collisionality profiles. They also found the coupling of the drift mode to the externally excited Alfvén waves, which resulted in frequency changes in the drift mode. Brochard et al. [197] investigated the nonlinear coupling of drift-wave fluctuation in the wavenumber spectrum and showed that intermittent structures in weakly developed drift-wave turbulence can be produced by mode coupling.

Drift-wave measurements with the new probe array were performed in Large Mirror Device-Upgrade (LMD-
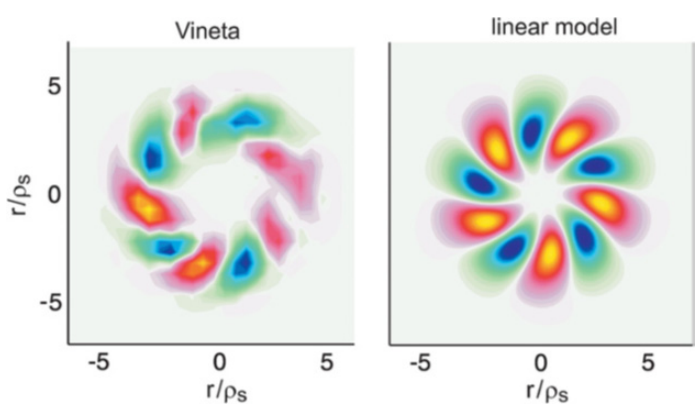

Fig. 18 Azimuthal density fluctuation pattern of a $m=5 \mathrm{drift}$ mode as measured experimentally (left) and as obtained from the linear dispersion relation model (right) [20].

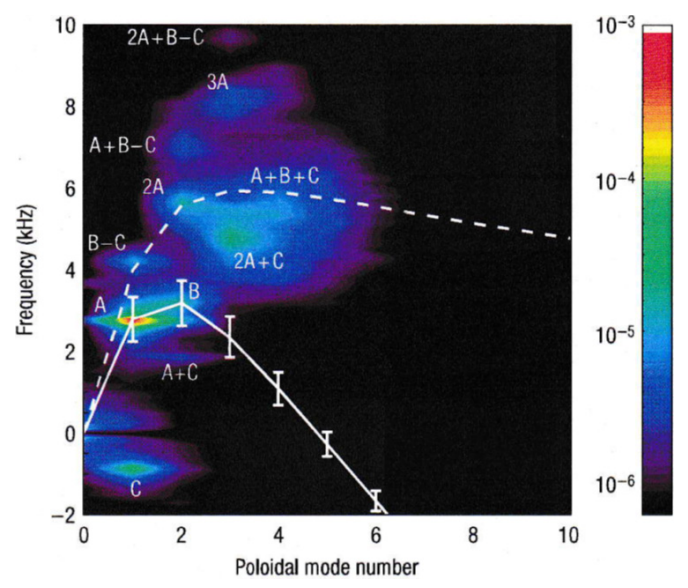

Fig. 19 Power spectrum $S(m, f)$ (arbitrary unit, contour) over plotted with the linear dispersion relation (solid line) calculated by a numerical simulation code. Error bars are standard deviations of several results using possible electron density profiles. The dashed line indicates the linear dispersion where the mean $E \times B$ drift motion is neglected. The calculated eigenmodes have positive growth rates [21].

U) [88]. Terasaka et al. [198] observed a change from the coherent to broadband density fluctuations by increasing the magnetic field. Figure 19 shows the two-dimensional power spectrum $[S(m, f)$, where $m$ is the poloidal mode number and $f$ is the frequency] of drift-wave turbulences in the helicon discharge measured by Yamada et al. [21]. The linear dispersion relation calculated by a numerical simulation code is also plotted with a white line in Fig. 19. The fluctuation modes $\mathrm{A}(m=1$ and $f=2.8 \mathrm{kHz})$ and $\mathrm{B}$ ( $m=2$ and $f=3.4 \mathrm{kHz}$ ) satisfy the linear dispersion relation of drift-waves. The peak at $m=1$ and $f=-0.9 \mathrm{kHz}$, which is considered a flute mode, is labeled $\mathrm{C}$, and all other peaks appearing in Fig. 19 can be expressed by the addition and/or subtraction of A, B, and C. Therefore, the formation of turbulences can be regarded as a result of the non-linear interaction of parent modes that satisfy the linear dispersion relation. 
Akhiezer et al. [199] suggest that the mechanism of ion-sound kinetic instability, which is excited by the parent helicon wave and the absorption of the ion-sound wave energy by electrons, can be a sufficiently strong mechanism for power absorption. Kline et al. [15, 16, 200] have observed the parametric decay of the helicon wave into two electrostatic waves. Figure 20 [16] shows the electric field power spectrum, and one may note the parent wave of $11 \mathrm{MHz}$, forward $\left(f_{0}+f_{1}\right)$ and backward $\left(f_{0}-f_{1}\right)$ propagating waves, and low-frequency beat wave (a spectral peak at approximately $9 \mathrm{MHz}$ is an experimental artifact). They also suggest that the TG wave and these parametrically excited waves are responsible for ion heating in the perpendicular direction [15, 16, 200, 201]. Figure 21 [15] shows the electron temperature, electron density, and perpendicular ion temperature as a function of antenna frequency and magnetic field strength. As seen from Fig. 21 (a) and (b), there is a clear increase in electron temperature as the antenna frequency nears the lower hybrid frequency. For antenna frequencies of $8-12.5 \mathrm{MHz}$, the largest plasma densities occur for $\omega \propto \omega_{\text {LH }}$. Figure 21 (c) shows the perpendicular ion temperature peaks for $\omega<\omega_{\mathrm{LH}}(0)$ (on axis)

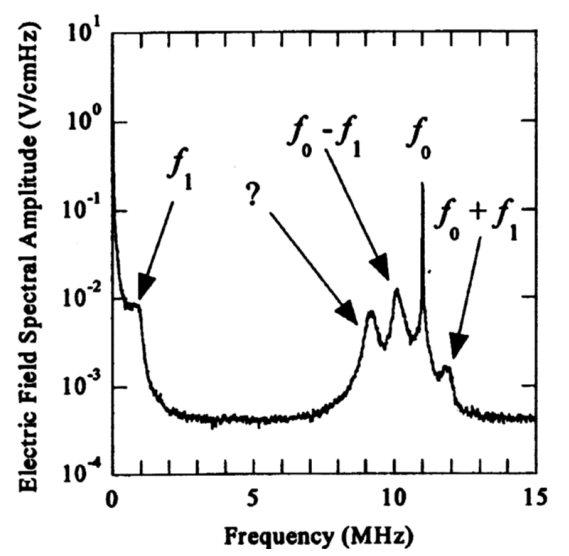

Fig. 20 Electrostatic fluctuation spectrum for a RF frequency of $11 \mathrm{MHz}$ and a magnetic field strength of $845 \mathrm{G}$ [16]. because of the low plasma density near the plasma edge (the local lower hybrid frequency drops below $10 \mathrm{MHz}$ ). The radial profile of the perpendicular ion temperature also shows the strong perpendicular heating at the plasma edge. This suggests the mechanism of the ion-Landau damping of the electrostatic TG waves at a local lower hybrid frequency resonance [15]. When the RF wave frequency equals the local lower hybrid frequency, the perpendicular wavenumber of the electrostatic TG wave goes to infinity. At a large perpendicular number, the wave phase velocity is sufficiently low that ion-Landau damping can occur. Lorenz et al. $[17,63]$ have demonstrated the fluctuations that originate from the parametric decay of the helicon waves into the ion-sound and TG waves, which showed good agreement of the measured growth rate and threshold with theoretical prediction for the parametric decay of helicon waves.

In this chapter, the recent studies on the instabilities in helicon discharge were overviewed. Drift-wave-type instabilities have been observed in linear helicon devices, which affect the plasma transport and equilibrium state of helicon discharge. The parametric decay instabilities of the helicon pump wave in the ion-sound and TG waves and the lower hybrid resonance effect of the TG waves have been suggested to play roles in efficient power coupling in helicon discharge. We need further investigations for these contributions on the power balance of helicon discharge.

\section{Other Experiments}

Shinohara et al. [58,202] have investigated the properties of helicon plasma by changing the wavenumber spectrum, different kinds of end plates [203], and the device aspect ratio [203] and in a very strong magnetic field up to $B_{0} \sim 10 \mathrm{kG}$ [3]. They have succeeded in controlling the plasma density profile by inserting the voltage-biasing electrodes [204,205] and changing the magnetic field configurations and antenna radiation field patterns [206].

Nisoa et al. [207-209] have produced high-density helicon plasma by exciting the $m=0$ standing helicon wave
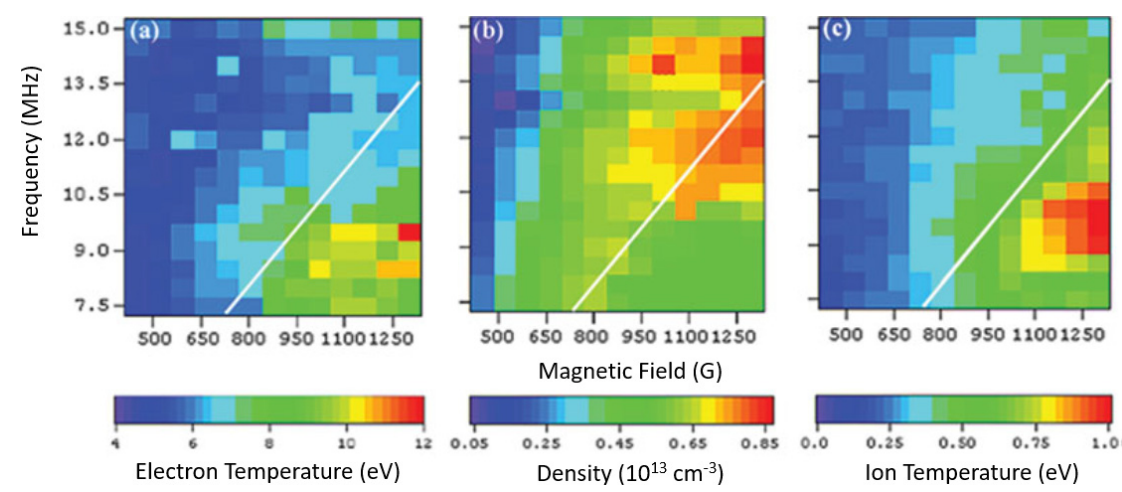

Fig. 21 (a) Electron temperature, (b) plasma density, and (c) ion temperature versus antenna frequency and magnetic field strength. The white line indicates where the antenna frequency equals the on-axis lower hybrid frequency [15]. 


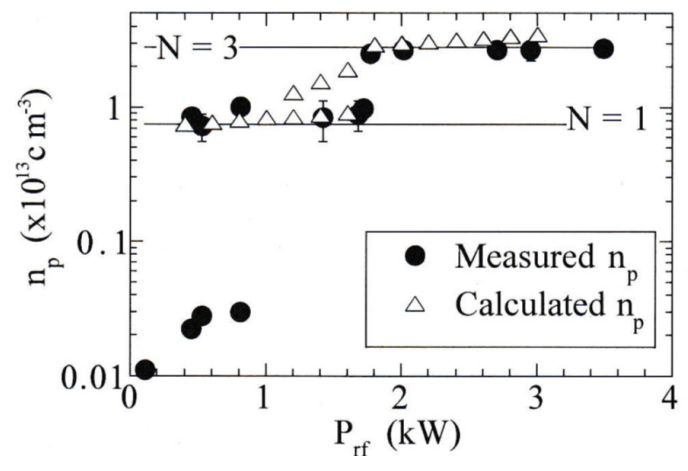

Fig. 22 Measured plasma density $n_{\mathrm{p}}$ at $B_{0}=237 \mathrm{G}$ and the calculated stable $n_{\mathrm{p}}$ versus $P_{\mathrm{RF}}$. Lines represent $n_{\mathrm{p}}$ at the peaks in plasma resistance for $N=1$ and 3 modes [209].

and observed the mode transition from $N=1(N$ is the number of half-wavelengths in the plasma column) to the $N=3$ standing wave mode as the input power (plasma density) increased. Figure 22 shows the measured plasma density $n_{\mathrm{p}}$ versus $P_{\mathrm{RF}}$, and stable $n_{\mathrm{p}}$ was calculated by using a 2D wave code (TASK/WF) [210]. The density jump can be seen clearly due to the mode transition from $N=1$ to $N=3$ at $P_{\mathrm{RF}} \sim 1.8 \mathrm{~kW}$. A low-density plasma $n_{\mathrm{p}} \sim 10^{11} \mathrm{~cm}^{-3}$, is thought be produced by the antenna induction field when $P_{\mathrm{RF}}<0.4 \mathrm{~kW}$, but no theoretical prediction is given to explain this low-density mode. In their calculation, there are two stable $n_{\mathrm{p}}$ that appear between the lines of the $N=1$ and 3 modes, and the observed densities always show agreements with the lower $n_{\mathrm{p}}$ values, but the reason for this is unclear. The calculated stable densities and the density jump point agree well with the measured ones.

Yun et al. [211, 212] have investigated the helicon plasmas near the lower hybrid frequency. They found that abrupt density profile changes occur near the lower hybrid frequency [212]. The centrally peaked density profile turns into the hollow density profile at particular frequencies. Figure 23 shows the measured frequencies when the hollow density profile is observed with various magnetic fields and ion masses. The theoretical lower hybrid frequency calculated using the measured density is also plotted in this figure. One may note that the measured frequencies have a correlation between the radial density profile and the lower hybrid frequencies.

Loewenhardt et al. [213, 214] have investigated helicon wave propagation in toroidal geometry and showed geometry influences on the propagation of waves by comparing the measured density with predictions from the helicon dispersion relation. Chang et al. [215] numerically studied the effects of axial gradient in plasma density and a static magnetic field on wave propagations by modeling the experiment in a magnetized plasma interaction experiment (MAGPIE) [216]. They showed the axial gradient in the magnetic field increased the decay length of helicon

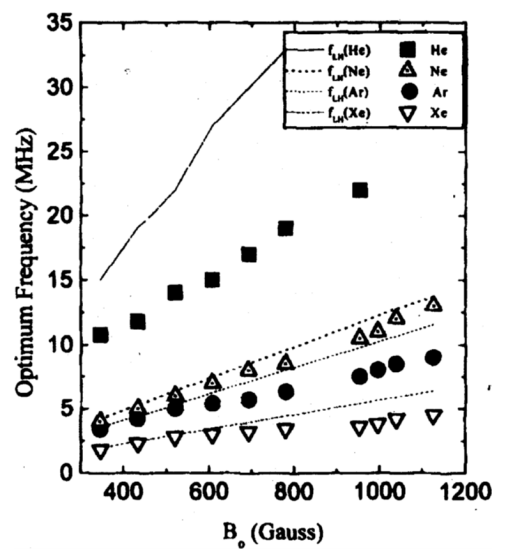

Fig. 23 Frequencies where the hollow density profile is observed with various magnetic fields and ion masses (at RF power $800 \mathrm{~W}$ and neutral pressure $3.5 \mathrm{mTorr}$ ) (dotted graph). The theoretical lower hybrid resonance frequencies for each ion are shown as a line graph [212].
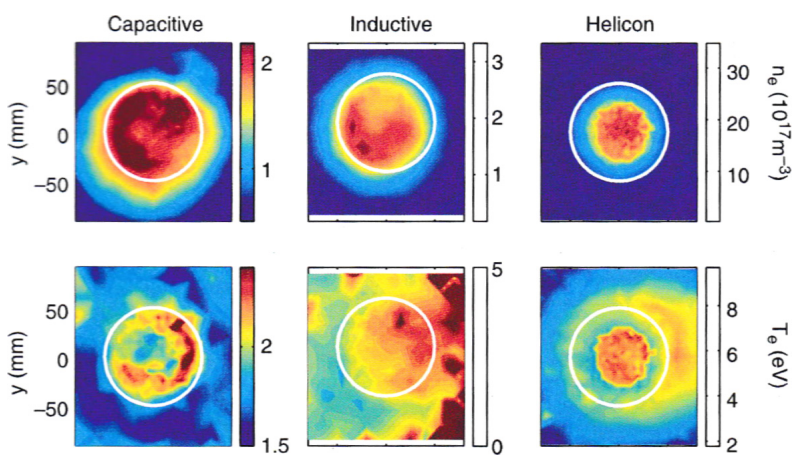

Fig. 24 Equilibrium plasma parameter profiles in the poloidal xy-plane perpendicular to the ambient magnetic field. Plasma density (top row) and electron temperature (bottom row) for all three discharge modes are shown. White circles indicate the position of the antenna edge [217].

waves in the downstream region, and the wave amplitude in non-uniform plasma density was much bigger in both the downstream and upstream regions than in the case of uniform plasma density. They also showed that the wave amplitude became lower and the power deposited into the core plasma decreased as the Coulomb collision frequency increased due to the strong edge heating.

Franck et al. [217] report on two-dimensional measurements of plasma parameters and magnetic eigenmode profiles in the capacitive-, inductive-, and helicon-wavesustained discharge modes of a helicon source with high spatial resolution. Figure 24 shows the plasma parameters of three discharge modes. The capacitive mode $\left(P_{\mathrm{RF}}=\right.$ $400 \mathrm{~W}, P_{\mathrm{Ar}}=0.08 \mathrm{~Pa}, B_{0}=56 \mathrm{mT}$ ): The plasma density is in the order of $10^{17} \mathrm{~m}^{-3}$, which is relatively high compared with the typical CCP discharge $\left(10^{15}-10^{16} \mathrm{~m}^{-3}\right)$ $[60,97]$, and has a peak of $n \sim 2.0 \times 10^{17} \mathrm{~m}^{-3}$ extend- 
ing from the edge region. The electron temperature profile is hollow, and peak values of $T_{\mathrm{e}} \sim 2.5 \mathrm{eV}$ are found at the antenna edge. The inductive mode $\left(P_{\mathrm{RF}}=600 \mathrm{~W}\right.$, $\left.P_{\mathrm{Ar}}=0.6 \mathrm{~Pa}, B_{0}=56 \mathrm{mT}\right)$ : The plasma density has an almost flat-top and the peak density $n \sim 3.0 \times 10^{17} \mathrm{~m}^{-3}$. The electron temperature follows the flat-top of the plasma density and has a peak value of $T_{\mathrm{e}} \sim 4 \mathrm{eV}$. The helicon mode $\left(P_{\mathrm{RF}}=3500 \mathrm{~W}, P_{\mathrm{Ar}}=0.5 \mathrm{~Pa}, B_{0}=75 \mathrm{mT}\right)$ : The plasma density and electron temperature are strongly peaked at the center of the plasma column with peak values of $n \sim 3.0 \times 10^{18} \mathrm{~m}^{-3}$ and $T_{\mathrm{e}} \sim 8 \mathrm{eV}$, respectively. They also showed differences between the magnetic eigenmode structures of the helicon and capacitive modes. The dereference between the inductive and helicon modes is not discussed in detail.

Corr et al. [218] investigated ion-acoustic wave phenomena in helicon sources. The wave amplitudes are spatially localized near the edge of a plasma column where there is a significant gradient in the plasma pressure as the amplitudes of the "mirror waves" that are separated from the RF frequency by the ion-wave frequency. Although the wave spectra are indicative of a parametric decay phenomenon, they suggest two possible mechanisms for the ion-wave excitation (i.e., the parametric decay and the radial pressure gradient).

Ganguli et al. [219] theoretically presented the damping and energy absorption for the helicon and TG wave modes, including both Landau and collisional damping inside a conducting waveguide. They also conducted the experiment $[219,220]$ with an axial magnetic mirror profile in two separate regimes. The first was a low-pressure $(\sim 0.2-0.3 \mathrm{mTorr})$ and low-magnetic-field $(\sim 16-20 \mathrm{G})$ regime where the Landau damping played a dominant role in the power absorption [219]. The second was a moderatepressure $(\sim 10$ mTorr $)$ and moderate-magnetic-field $(\sim 60$ $65 \mathrm{G})$ regime. In the first case, both the wave absorption and warm electron production were shown to proceed through Landau damping. In the second case, where the power absorption was collisional, they observed a double layer with large potential drops [221], and warm electron production proceeded via high-energy electrons produced by the acceleration of bulk electrons (from neighboring regions) across large potential gradients.

Breizman and Alexey [222] suggest that the radial density gradient can create a radial potential well for nonaxisymmetric whistlers, allowing radially localized solutions with a slightly lower frequency shift, which is called a radially localized helicon (RLH) wave. Panevsky and Bengtson [223], Chen et al. [224], and Lee et al. [225] showed evidence for the existence of the RHL wave experimentally and numerically.

\section{Remaining Critical Issues}

Since helicon studies began in 1960, many parts of helicon plasma physics have been clarified owing to numer- ous theoretical and experimental attempts. However, the exact mechanism responsible for high-efficiency plasma production is not yet completely understood. In recent studies, many new physical mechanisms, such as driftwave instability and parametric decay instability, have been successfully revealed. These mechanisms affect plasma production and diffusion transport. Therefore, the helicon source should be optimized to include these effects more precisely. Here, we summarize the critical remaining issues.

(1) The mode transition from the H-mode to the W-mode

The density jump between the intermediate-density mode to the high-density mode is believed to be a mode transition from the ICP $(\mathrm{H})$ mode to the helicon (W) mode. However, as mentioned in Sec. 6, the dispersion relation predicts that the TG wave will propagate even at a lowdensity, and can contribute to the RF power deposition. Actually, the induction fields observed at a low-density in the experiments $[47,48,57,59]$ are evanescent helicon waves, which oscillate near the antenna (within their skin depth). Therefore, which wave is responsible for RF absorption should be investigated. Indeed, the low-density plasmas sustained by the TG waves are observed in a low magnetic field [92] and very high drive frequency range [107], where the wavelength of the TG wave becomes comparable to the plasma radius. The difference between the ICP mode and the helicon mode should be investigated in more detail from the perspective of the power balance.

(2) Density limit in helicon discharge

The major factor that limits the density of helicon discharge seems to be neutral depletion [24]. The mechanism of neutral depletion should be considered with two main parameters, the collision frequency and the magnetic field. In collisionless plasma, neutral depletion occurs only due to electron impact ionization. In collisional plasma, the total pressure balance between the neutral and plasma results in neutral depletion due to the drag force of ion-neutral collision. In high-beta plasma, the total pressure balance should contain the magnetic pressure that results from the diamagnetic effect [151]. Besides these parameters, the ion and neutral temperatures also affect the drag force (the collision frequency) and pressure balance. More detailed investigations are needed both theoretically and experimentally to overcome the density limit.

\section{(3) Centrally peaked density profile}

Diffusion across the magnetic field remains a controversial issue. Chen et al. $[6,152,168]$ insist that the shortcircuit effect plays a crucial role in the centrally peaked density profile. On the other hand, the drift-wave turbulences observed in the helicon source [19-21,192-196] can also be a candidate for anomalous diffusion. There is no consensus on which plays a dominant role in plasma transport across the magnetic field. A self-consistent model which includes the instabilities and enhanced plasma trans- 
port, is needed for an improved understanding.

\section{(4) Instabilities}

The saturation and decrease of plasma density above the critical values of the magnetic field seem to be caused by the drift-wave instability $[18,82]$. The effect on the particle balance and plasma transport should be investigated in more detail. The non-linear RF power coupling caused by the parametric decay instability can also be a strong mechanism for power absorption [199]. The relative importance of the power absorption mechanism should be discussed in relation to normal joule heating.

\section{(5) Ion heating}

The heating of perpendicular ion temperature has been observed in experiments $[15,16,200,201]$. The TG wave and these parametrically excited waves are responsible for ion heating. The ion-Landau damping caused by the lower hybrid resonance TG waves seems to be the main mechanism of the ion heating. The ion heating also affects neutral depletion and plasma transport. Therefore, the ion dynamics and energy balance should also be included in the helicon discharge model.

\section{Attempts for Self Consistent Model}

The remaining issues in Sec. 11 are closely related; for example, ion or neutral heating enhances neutral depletion, which leads to enhanced plasma transport and the dramatic change of the power balance and density profile, and this determines the density limit in helicon discharge. Furthermore, the effects of instabilities may critically affect all these processes. In addition, the power absorptions from the RF antenna via the electrostatic (the $\mathrm{E}$ mode) and the electromagnetic fields (the $\mathrm{H}$ and $\mathrm{W}$ modes) and plasma transport processes should be treated self-consistently. There are various timescales in helicon discharge and each physical parts affects the other in the time evolution of helicon discharge.

Figure 25 shows the timescales in helicon discharge, which are evaluated by using the typical parameters in HELIX [15]. The upper panel shows the time periods of the drive frequency $T_{\mathrm{RF}}=2 \pi / \omega_{\mathrm{RF}}$, the electron (ion) cyclotron frequency $T_{\text {ce(ci) }}=2 \pi / \omega_{\text {ce(ci) }}$, the lower hybrid frequency $T_{\mathrm{LH}}=2 \pi / \omega_{\mathrm{LH}}$, and the collision periods of electron-neutral $\tau_{\text {en }}$, electron-ion $\tau_{\text {ei }}$, electron-electron $\tau_{\text {ee }}$, ion-neutral $\tau_{\text {in }}$, ion-electron $\tau_{\text {ie }}$, and ion-ion $\tau_{\text {ii }}$. These collision frequencies are given in $[226,227]$. The bottom panel shows the times scales of the axial diffusion $\tau_{\|, \mathrm{e}}=$ $L_{z}^{2} / D_{\|, \mathrm{e}}$ and perpendicular diffusions of $\tau_{\perp, \mathrm{i}}=L_{z}^{2} / D_{\perp, \mathrm{i}}$, and $\tau_{\perp, \text { Bohm }}=L_{z}^{2} / D_{\perp, \text { Bohm }}$, where $D_{\|, \mathrm{e}}, D_{\perp, \mathrm{i}}$, and $D_{\perp, \text { Bohm }}$ are the diffusion coefficients of the electrons along the field line, perpendicular ion diffusion, and Bohm diffusion, respectively. Here, we consider non-classical diffusions, such as the short-circuit effect and anomalous diffusion. The ionization timescale is evaluated as $\tau_{\text {ion }}=1 / n_{\mathrm{g}} \alpha_{\text {ion }}$, where $\alpha_{\text {ion }}$ is the ionization rate [226]. The energy confinement timescale is evaluated as $\tau_{\text {ene }}=n T_{\mathrm{e}} V / P$, where $V=\pi L_{r}^{2} L_{z}$ is the plasma volume. The growth timescale of the drift-wave $\tau_{\mathrm{dw}}$ is estimated by solving Ellis's equations $[75,194]$ with the Gaussian parameter $a^{2} / r_{0}^{2}=1.75$ (where $a$ is the plasma radius and $r_{0}$ is the scale length of the Gaussian), azimuthal mode number $m=1$, axial wavelength $\lambda_{\|}=5 \mathrm{~m}$, and collision frequencies in Fig. 25 . Since the drift-wave becomes unstable for the long axial wavelength [75], the axial wavelength $\lambda_{\|}=5 \mathrm{~m}$ is used here. This wavelength is larger than $L_{z}=1.6 \mathrm{~m}$, but this is reasonable since the HELIX is combined with a large, 2$\mathrm{m}$-diameter, 4-m-long space simulation chamber (LEIA)

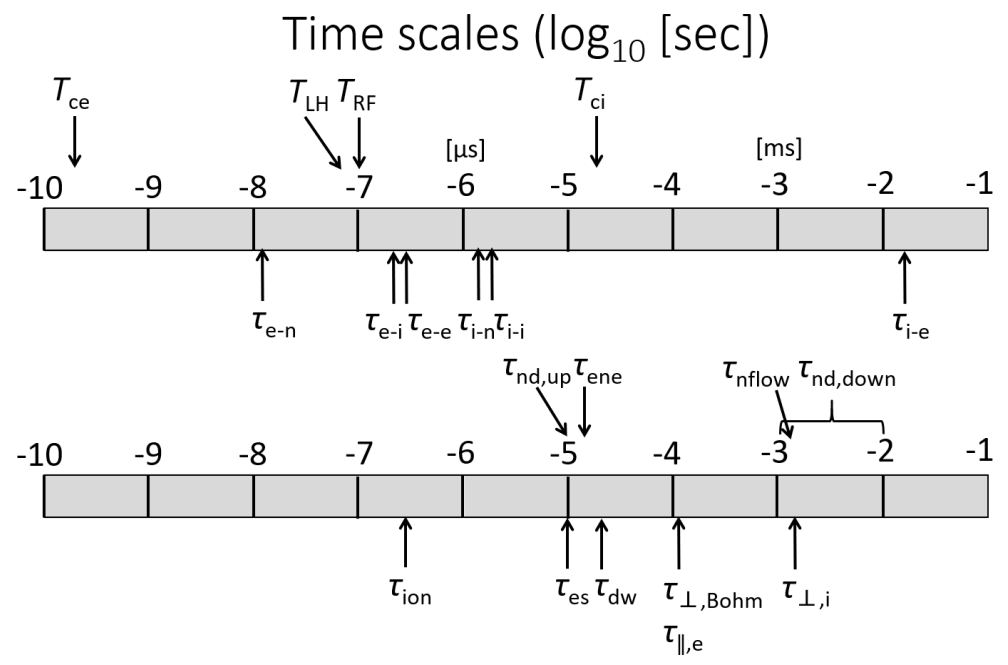

Fig. 25 Various physical time scales with following typical parameters in HELIX [15]; plasma density $n=2.0 \times 10^{18} \mathrm{~m}^{-3}$, electron temperature $T_{\mathrm{e}}=8.0 \mathrm{eV}$, ion temperature $T_{\mathrm{i}}=0.5 \mathrm{eV}$, RF frequency $f_{\mathrm{RF}}=10 \mathrm{MHz}, \mathrm{RF}$ power $P_{\mathrm{RF}}=750 \mathrm{~W}$, magnetic field $B_{0}=0.1 \mathrm{~T}$, Ar pressure $P_{\mathrm{Ar}}=6.7 \mathrm{mTorr}$, source tube radius $L_{r}=0.075 \mathrm{~m}$, and axial length $L_{z}=1.6 \mathrm{~m}$. 
[147]. The growth timescale of the electrostatic (ES) fluctuation $\tau_{\mathrm{es}} \sim 10^{-5} \mathrm{~s}$ was observed by Krämer et al. [17]. The neutral depletion has a range of timescales: slow depletion, the order of $\tau_{\text {nd,down }} \sim 10 \mathrm{~ms}$, was observed in the downstream region from the antenna, and fast depletion, the order of $\tau_{\text {nd,up }} \sim 10 \mu \mathrm{s}$, which showed significant depletion of $1 \%$ of its initial value, was observed near the antenna in Magee's experiment [25]. The short timescale depletion of $\tau_{\text {nd,down }} \sim 1 \mathrm{~ms}$ was observed in the downstream region in Liard's experiment [133]. Magee et al. [25] insisted that the fast neutral depletion $(\sim 10 \mu \mathrm{s})$ observed near the antenna resulted from the ion-pumping (ionization) effect rather than the neutral-pumping effect. The neutral inflow timescale $\tau_{\text {flow }}$ was estimated to be the traveling timescale of the krypton neutral from the gas inlet to $30 \mathrm{~cm}$ with thermal velocity [25].

Since the RF period $T_{\mathrm{RF}}$ is much faster than the other timescales, the timescale of the TG and helicon wave perturbations are well separated from those dynamics. Therefore, the wave fields can be treated as steady parts. The timescale of the ionization $\tau_{\text {ion }}$ is also well separated, and the rapid growth of the plasma density can be treated separately. However, the relaxation timescale for the steadystate plasma density should be determined from the plasma diffusion timescales. After the density grows near the antenna, the plasma density expands to the axial diffusion chamber in the timescale of $\tau_{\|, \mathrm{e}} \sim 0.1 \mathrm{~ms}$ (with axial length $L_{z}=1.6 \mathrm{~m}$ ), and the radial plasma density profile relaxes to the steady-state in the timescale of $\tau_{\perp, \mathrm{i}} \sim 2 \mathrm{~ms}$ (with radial length $L_{r}=0.075 \mathrm{~m}$ ). Indeed, the plasma density observed at the expansion chamber relaxed to the steadystate in the timescale of $2-5 \mathrm{~ms}[17,25]$. While the plasma density relaxes to the steady state, the ES and drift-wave instabilities occur within the timescale of $\sim 20 \mu \mathrm{s}$, and these instabilities affect the particle and energy balances via the non-linear power absorption and amorous diffusions, respectively. Therefore, the plasma diffusion, energy balance (the energy confinement time is also in the same order as the growth timescales of the instability), and these instabilities should be treated self-consistently. The neutral depletion can be considered separately in the upstream region (near the antenna) and in the downstream region. The former may result from the ion-pumping effect in the fast timescale of $10 \mu \mathrm{s}$, and latter may result from the neutralpumping effect in the slow timescale of $1-10 \mathrm{~ms}$. Regarding the neutral depletion in the downstream, the pressure balance between neutral and plasma should be treated selfconsistently with the plasma diffusion. Since the neutral depletion timescale that results from the ion-pumping effect is much shorter than the neutral inflow timescale, the gas inlet position should be close to the antenna to overcome the significant neutral depletion near the antenna. The neutral depletion that results from the pressure balance would be improved by controlling the pressure balance by applying the inhomogeneous magnetic field or applying the diamagnetic effect.
The helicon discharge system is so complicated that we cannot easily include these physical parts selfconsistently, and this attempt is challenging for developing the next-generation helicon source. Several basic selfconsistent models of helicon discharge have been developed.

Kinder et al. have developed the two-dimensional model [228,229] of the Trikon PMT MØRI helicon source [230] and three-dimensional model [231] of the Wave On Magnetized Beams and Turbulence (WOMBAT) helicon source [84, 232] for use in material processing. Their hybrid plasma equipment model (HPEM) [228, 229, 233] calculates the wave fields excited by the RF antenna, electron energy equation in which power absorption by wave fields and power loss due to collision is included, and fluid continuity, momentum, and energy equations for ion and neutrals (the drift-diffusion formation in which the inertial term is omitted is used for the electron momentum equation). These calculations are iterated until a converged solution is obtained. This model also includes the Electron Monte Carlo Simulation (EMCS) for calculating the electron energy distribution (EED). They showed the differences between the $\mathrm{H}$ mode (ignoring the TG wave), the $\mathrm{W}$ mode, and the TG wave-sustained mode.

The left panel of Fig. 26 [229] shows azimuthal, radial, and axial electric field profiles and power absorption profiles at $20 \mathrm{G}$ and $300 \mathrm{G}$ for the $\mathrm{H}$ and $\mathrm{W}$ modes, respectively, where the TG wave is omitted in this calculation. As shown in Fig. 26, at $B=20 \mathrm{G}$, the azimuthal electric field is predominantly inductively coupled with an enhanced plasma skin depth. At $B=300 \mathrm{G}$, the electric field profiles show the traveling wave (the helicon wave),

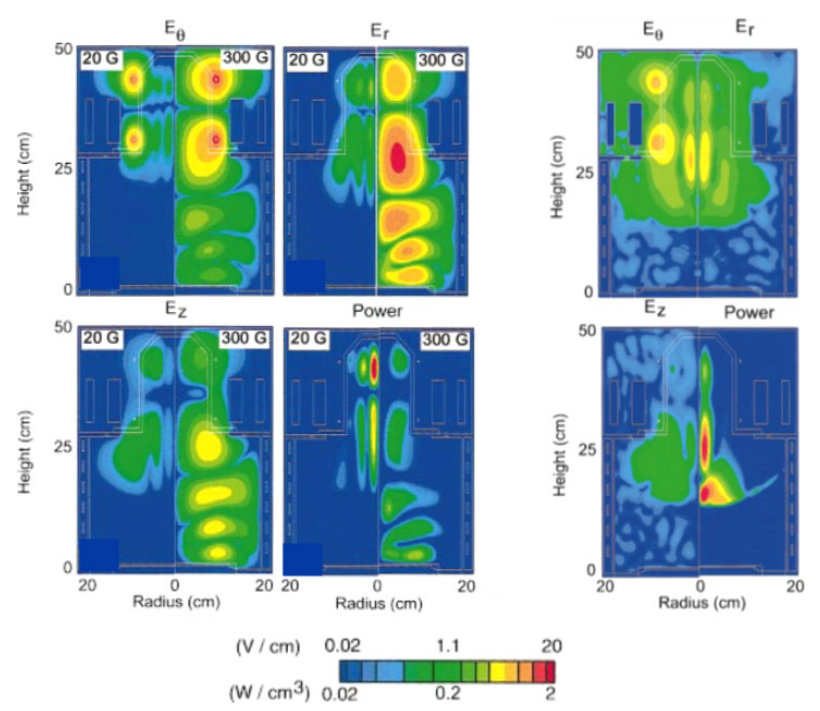

Fig. 26 The electric field profiles and power absorption profile of the $\mathrm{H}\left(B_{0}=20 \mathrm{G}\right)$ and $\mathrm{W}$ modes $\left(B_{0}=300 \mathrm{G}\right)$ without the TG wave (left panel) and low-magneticfield $\left(B_{0}=20 \mathrm{G}\right)$ operation with including the TG wave (right panel) [229]. 
which propagates to the downstream. At $B=20 \mathrm{G}$, the power deposition peaks at $2.1 \mathrm{~W} / \mathrm{cm}^{3}$, and at $B_{0}=300 \mathrm{G}$, the same total power is deposited over a large volume, resulting in a lower peak power deposition. The left panel of Fig. 27 [229] shows the electron temperature $\left(T_{\mathrm{e}}\right)$ and the plasma density (e) profiles for the $\mathrm{H}(B=20 \mathrm{G})$ and $\mathrm{W}$ modes $(B=300 \mathrm{G})$. At $B=20 \mathrm{G}$, the electron temperature and electron density peak at $\sim 4 \mathrm{eV}$ and $4 \times 10^{12} \mathrm{~cm}^{-3}$ near the antenna, respectively, where most power disposition occurs. At $B=300 \mathrm{G}$, the electron temperature and electron density peak at $\sim 3.5 \mathrm{eV}$ and $3.2 \times 10^{12} \mathrm{~cm}^{-3}$, respectively in the downstream. They also show that the tail of the EED increases in magnitude in the downstream chamber for $B_{0}>150 \mathrm{G}$ and $P<5$ mTorr, which indicates some electron trapping and collisionless heating with the phase matching of thermal electrons to the phase velocity of the wave.

The right panel of Fig. 26 shows the electric field profiles and power absorption profile including the TG wave at a low magnetic field of $B_{0}=20 \mathrm{G}$. In this case, the radial wavelength of the TG wave is comparable to the plasma size; therefore, the TG wave, which propagates into the core of the plasma, can be seen. Although the power absorption peaks near the location of the peak electric fields in the upstream region, significant power deposition also occurs in the electron cyclotron (ECR) zone where the drive frequency $f_{\mathrm{RF}}=13.56 \mathrm{MHz}$ matches the electron cyclotron frequency at $B_{0}=4.8 \mathrm{G}$. The right panel of Fig. 27 shows the electron density perturbation and the electron density profiles at $B_{0}=20 \mathrm{G}$ and $B_{0}=300 \mathrm{G}$ including
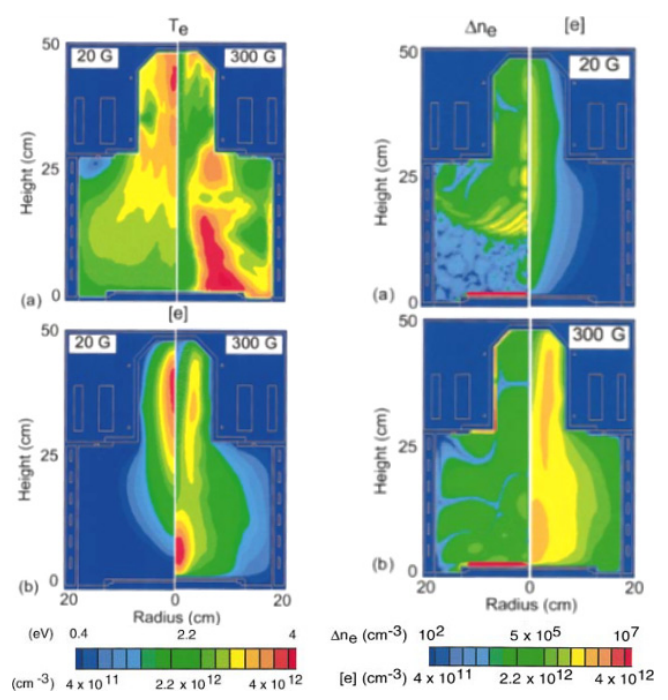

Fig. 27 Left panel: electron temperature and plasma density profiles of the $\mathrm{H}\left(B_{0}=20 \mathrm{G}\right)$ and $\mathrm{W}$ modes $\left(B_{0}=\right.$ $300 \mathrm{G}$ ) without the TG wave. Right panel: electron density perturbation $\left(\Delta n_{\mathrm{e}}\right)$ and electron density (e) profiles at low-magnetic-field $\left(B_{0}=20 \mathrm{G}\right)$ and high-magneticfield $\left(B_{0}=300 \mathrm{G}\right)$ operations including the TG wave [229]. the TG wave. At $B_{0}=20 \mathrm{G}$, the electrostatic term can be seen through the harmonically driven perturbation in the electron density, and there is significant propagation into the chamber until the ECR zone appears. Therefore, the low-density (the peak value of $\sim 2.8 \times 10^{12} \mathrm{~cm}^{-3}$ ) mode is sustained by the TG wave. At $B_{0}=300 \mathrm{G}$, in this case, the wavelength of the TG wave is so short that the TG wave is strongly absorbed near the plasma surface. In this case, although the electron density profile appears similar to that of the pure helicon case, the average electron density of $7.7 \times 10^{11} \mathrm{~cm}^{-3}$ is higher than that of the pure helicon case $\left(2.4 \times 10^{11} \mathrm{~cm}^{-3}\right)$. These results provide evidence of the low-density TG wave-sustained mode in the low magnetic field, which is observed in Lho's experiment [92], and show the clear differences in the power absorption, plasma density and electron temperature profiles between the $\mathrm{H}$-, $\mathrm{W}$-, and TG wave-sustained modes.

By using the same model, Bose et al. [234] showed that edge heating due to the TG waves dominated the power absorption under high external magnetic fields. Cho et al. [235] also studied the effect of the external magnetic field on the radial plasma density profile with the onedimensional steady-state fluid model combined with wave equations. They showed that the plasma density changed from a relatively flat to steep profile as the external magnetic field increased, and when the TG wave dominated the power absorption, the density profile was relatively flat in the central region compared with the case when there was significant bulk heating by the helicon wave. In their results, the linear dependence of the plasma density on the magnetic field, which is one of the typical characteristics observed in helicon discharges, was also demonstrated. Chen et al. [236] used the two-dimensional steadystate fluid model of Boswell's experiment [1]. They found that the classical heat conductivity was too small to sustain a centrally peaked density profile under the experimental conditions, and the resulting density profile showed a hollow density profile. Assuming enhanced radial heat conductivity and particle transport, they also found steadystate solutions that were in good agreement with density profile and density jump observed in the experiment. Isayama et al. [237] first discussed the temporal behavior of the helicon discharge with the time-integrated fluid model combined with the wave equations calculated by the finite-difference time-domain (FDTD) method. They showed the two time phases in the time evolution of the helicon discharge. In the early stage of discharge growth, the TG wave dominated the power absorption near the plasma edge region; thus, the edge-localized electron temperature and plasma density profile grew over time. After the density grew sufficiently for the helicon wave to be able to propagate, the helicon wave deposited its power in the core of the plasma. Eventually, the centrally peaked density profile was obtained in the steady-state. The combination of these self-consistent discharge models with instability models and neutral dynamics is a challenging issue for a 

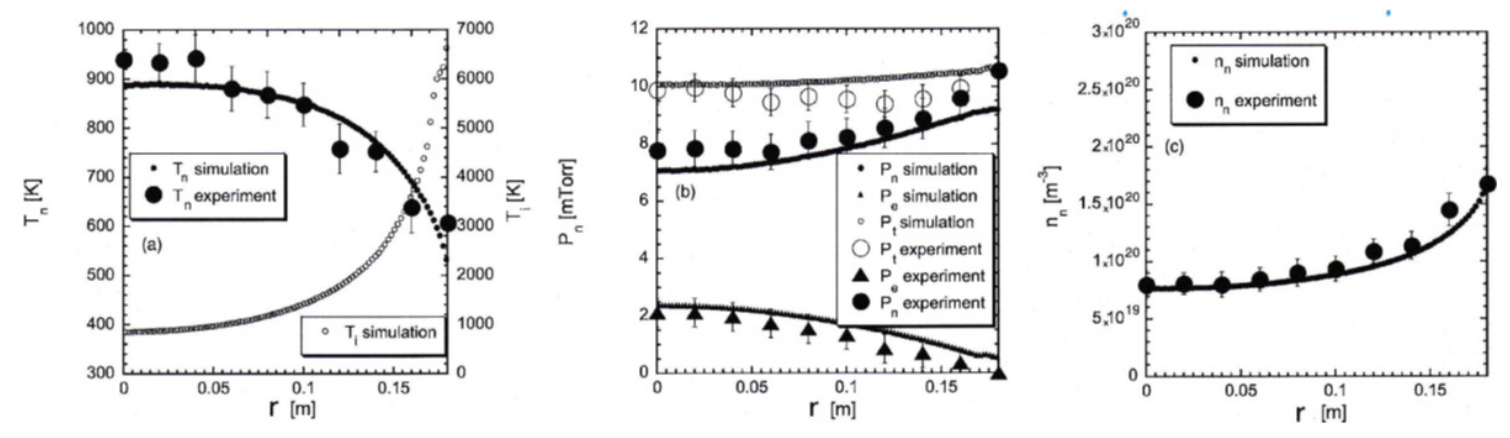

Fig. 28 Comparison of the numerical and the experimental results. (a) Neutral and ion temperature profiles, (b) neutral, electron and total pressure profiles, and (c) neutral density profile [119].

through understanding of helicon discharge. There are several models of drift-wave instability and neutral dynamics.

Numerical simulations of the drift-wave turbulence in linear devices have been carried by Kervalishvili et al. [238] and Kasuya et al. [239-241]. Kervalishvili et al. [238] modified the original three-dimensional Gyrofluid Electro Magnetic (GEM3) code [242] for cylindrical annulus geometry to use it for the simulation of VINETA. They showed the linear growth of the potential fluctuation and found strong peaks for the $m=6$ mode, which corresponds with the experimentally observed fluctuations of $m=1$ 8 [193]. Kasuya et al. [240] developed a three-dimensional numerical simulation code for the resistive drift-wave turbulence in a linear device, called the Numerical Linear Device (NLD). The selective formation of the zonal flow and the streamer using ion-neutral collision $v_{\text {in }}$ as a control parameter was observed in their simulation results. The value of $v_{\text {in }}$ represents the strength of the damping force of the turbulent structures. When the collision frequency is large, the zonal flow remains stable and the streamer is formed. The bifurcation of these structures was also investigated in their model. Chang et al. [243] applied a twofluid model to interpret plasma oscillations in the WOBAT device. They showed that the density and space potential profiles agreed with their experimental results, but the temperature profile was not flat in the observed results, as assumed by the model.

The direct simulation Monte Carlo (DSMC) method [244] is often used for computing the neutral gas distribution. Hartig and Kushner [245] and Kilgore et al. [246] investigated the neutral transport in ECR plasma. The neutral density was found to be depleted in the volume of the chamber due to ionization (ion-pumping), and the depletion was enhanced by neutral heating by electronneutral and ion-neutral collisions [246]. The combination model of the DSMC method with the particle-incell (PIC) method was developed by Vladimir et al. [247] for the DC and ICP discharges. Appreciable gas heating and depletion in the discharge was demonstrated. Shimada et al. [119] developed a hybrid-type DSMC model for the ICP discharge, in which electrons were treated as background particles using the measured electron density and electron temperature profiles, and ions and neutrals were treated as test particles. In this model, ionization, electron-ion, electron-neutral elastic, ion-neutral elastic, ion-neutral charge exchange, and neutral-neutral elastic collisions [244, 248] were all considered. Figure 28 [119] shows the comparison of the numerical and experimental results $[249,250]$ for (a) neutral $\left(T_{\mathrm{n}}\right)$ and ion $\left(T_{\mathrm{i}}\right)$ temperature profiles; $(\mathrm{b})$ neutral $\left(P_{\mathrm{n}}\right)$, electron $\left(P_{\mathrm{e}}\right)$, and total pressure $\left(P_{\mathrm{t}}\right)$ profiles; and $(\mathrm{c})$ the neutral density profile $\left(n_{\mathrm{n}}\right)$. Excellent agreement between the experiment and the simulation for the radial profiles of $T_{\mathrm{n}}, P_{\mathrm{n}}, P_{\mathrm{e}}, P_{\mathrm{t}}$, and $n_{\mathrm{n}}$ can be seen, and these results indicate that the neutral heating and pressure balance (neutral-pumping) cause the neutral depletion in ICP discharge.

\section{Applications}

Microprocessing is necessary for realizing the highdensity integration of semiconductor devices and the high performance of thin-film transistor liquid crystal panels [251]. One of the techniques for achieving this is a dry process, in which high-density plasma sources with an electron density of $10^{11}-10^{13} \mathrm{~cm}^{-3}$ are applied. The standard systems are CCP and ICP sources. Helicon sources would generate higher plasma density, which enables high deposition and etch rates. In the 90s, many helicon sources [230,252-255] were built for commercial use, but the issue of the uniformity of the plasma density remained, which is needed for uniform etching. In recent studies, Chen et al. $[6,256]$ have developed multiple helicon sources and successfully produced good uniform plasma with a density of up to $10^{12} \mathrm{~cm}^{-3}$. They have also developed a compact helicon source using a permanent magnet that is expected to be a more practical helicon source in the semiconductor industry.

The large helicon source has been used for a spacecraft intended for travel to Mars, the Variable Specific Impulse Magnetoplasma Rocket (VASIMR) [257]. In this system, the generated plasma is energized further by an ion cyclotron heating (ICH) RF stage [258] that uses left- 
hand polarized slow-mode waves launched from the high field side of the ion cyclotron resonance, and useful thrust is produced as the plasma accelerates in an expanding magnetic field [259]. In recent studies, the thruster efficiency and thrust power of a high-power VASIMR prototype have been measured at a level of $200 \mathrm{~kW}$ RF input power [258, 260-263].

The permanent magnet helicon current-free doublelayer (DL) thruster (PM-HDLT) has been developed by Takahashi [144-146, 264-271], Charles [272-275], Fruchtman [113,276], and their research team. The magnetic nozzle constitutes the acceleration stage of the helicon thruster, located in the downstream of the chamber. Theoretical analyses of the helicon plasma thrust performance with the magnetic nozzle have been developed [113, 276-278], and direct thrust measurements have also been performed in a number of devices [266-268, 279-282]. In recent studies, the performance has been improved by modifying the magnetic field configuration and increasing the magnetic field strength $[144,266]$. Takahashi et al. [269, 271, 283] point out that the neutral depletion induces an axial asymmetric density profile, which causes the decrease in the axial thrust.

A new concept of electrodeless discharge and electromagnetic acceleration has been developed by the helicon electrodeless advanced thruster (HEAT) project [110, 280, 284-295]. The electrodeless configuration is a promising solution for the erosion and contamination problems in conventional electric propulsion systems [284]. The rotating RF electric field (REF) [280, 285-288, 295] and rotating RF magnetic field (RMF) [110,289,291-293] are based on the concept that the entire acceleration process can be conducted without contacts between the electrodes and the plasma by using RF antennas outside of the discharge chamber. In these methods, an azimuthal electric current is induced by the additional REF or RMF, and the plasma is electromagnetically accelerated in the magnetic nozzle. Ponderomotive acceleration (PA) with the ion cyclotron resonance (ICR) acceleration method [290, 294] may efficiently heat ions perpendicularly through ICR, and, by applying the RF waves in such a way that the resonance point coincides with the peak of the wave energy density, the ions can gain parallel acceleration because of the EM ponderomotive force. The idea of the $m=0$ acceleration method $[4,289]$ is to induce an azimuthal current in the presence of the divergent magnetic field to produce the $J_{\theta} \times B_{r}$ axial force, like in the RMF and REF schemes. In a half-RF wave period (in the following half-period), a positive (negative) axial force is expected, which leads to a zero net force. Then, if the field driven by the antenna is sufficiently strong to push the plasma out of the acceleration region during one half-period, a net positive force remains.

In the nuclear fusion field, Loewenhardt et al. [34] and Zhang et al. [214] have investigated the characteristics of the toroidal helicon sources in the Small Heliac Experi- mental Apparatus (SHEILA) [296] and the HEALIC (H1) [297]. Shats et al. [298] report the sudden transition from the low confinement mode to the high confinement mode ( $\mathrm{L}-\mathrm{H}$ transition) in the $\mathrm{H}-1$ heliac and showed a clear dependence on magnetic configuration. They showed that the ion temperature increased from 40 to $80 \mathrm{eV}$ in this transition [299], and suggest the mechanism driving the radial electric field $E_{r}$ at a critical value before the bifurcation [300]. Tripathi et al. [301, 302] have investigated the characteristics of the helicon wave in a tight-aspectratio toroidal device and showed the significant difference between the toroidal mode and the conventional helicon mode.

For the study of the Earth's magnetosheath, Scime et al. and their research team [7, 147] have investigated the magnetohydrodynamic waves in HELIX-LEIA. The excellent agreement between the measurements and the theoretical prediction provide strong evidence of the successful excitation of Aflven waves [303].

\section{Concluding Remarks}

In this review, we have performed an extensive review of discussions on helicon plasma production mechanisms and introduced them in some detail with particular emphasis on physical processes involving helicon and TG waves.

In Secs. 1 and 2, the outline and brief history of the helicon study are described. Research on helicon plasma has revealed important mechanisms of efficient plasma production since helicon studies began in the $60 \mathrm{~s}$, when the observation of the helicon wave in metallic sodium was first announced.

In Sec. 3, the dispersion relation of the helicon wave is reviewed. In past decades, the mechanism of helicon plasma production has been investigated based on the dispersion relation of the helicon wave, ignoring the effect of electron inertia (the TG wave) $[1,87]$. The comparison with the experimental results shows good agreement with the dispersion relation $[1,59,92]$ and the wave profiles $[1,86,88]$.

In Secs. 4 - 6, the role of the TG wave and the density jump mechanism are reviewed. Since the role of electron inertia was fully recognized [41], efficient RF power coupling in helicon discharge has been understood with the collisional dissipations of the helicon and TG waves. The TG wave deposits its energy near the plasma surface [9-11], while the helicon wave penetrates into the core region and deposits its energy in the core plasma $[9,12]$. The resonance and anti-resonance properties of the TG wave excitation in the bounded cavity result in the nonmonotonic power dependence on the plasma density and the magnetic field [43]. Some parts of the density jump observed in the experiments $[1,59]$ can be explained based on the power balance with non-monotonic power absorption curves [43, 101, 209]. However, the discharge mode before the mode transition into the helicon mode (W) re- 
mains controversial issue. This low-density mode before the density jump is believed to be the ICP $(\mathrm{H})$ mode. When the finite magnetic field is applied, the ICP mode is precluded, which could be a low-density TG wave-sustained mode. Indeed, the low-density plasmas sustained by the TG waves are observed in a low magnetic field [92] and at a very high drive frequency range [107]. The induction fields observed at a low density in the experiments $[47,48,57,59]$ are evanescent helicon waves, which oscillate near the antenna (within their skin depth). Therefore, which wave is responsible for RF absorption should be determined.

In Sec.7, the neutral depletion mechanism is reviewed. Neutral depletion is considered to be responsible for the limitation of the plasma density in the helicon source [5,24,25]. There are two ideas for overcoming neutral depletion. One is the proper design of the neutral pump. The gas inlet position should be close to the antenna to overcome significant neutral depletion due to the ionpumping effect (ionization) near the antenna. The other is applying the inhomogeneous magnetic field or using the diamagnetic effect $[142,151]$. Neutral depletion resulting from the neutral-pumping effect (pressure balance) would be improved by controlling the pressure balance between the plasma pressure, the neutral pressure, and the magnetic pressure.

In Secs. 8 - 10, the plasma diffusion mechanism, nonlinear mechanisms, and other notable experiments are reviewed. There are two main mechanisms for the anomalous diffusion across the magnetic field in helicon discharge. One is the "short-circuit effect" [170], in which the ions can diffuse freely across the magnetic field without the restriction of ambipolarity, while electrons do not follow the ions across the magnetic field, but instead travel along the field lines and conducting wall, and eventually maintain charge neutrality $[152,168,172]$. The other is Bohm diffusion [22], which is predominantly interpreted as an upper limiting value of cross-field diffusion with lowfrequency drift-wave fluctuation. However, there is no current consensus on which theory to use to describe diffusion transport. Recent studies have revealed the considerable non-linear mechanisms of the drift-wave [18-21] and parametric decay $[15,17]$ instabilities. These non-nonlinear dynamics can affect the plasma transport and power balance. Other experiments have revealed various remarkable features of helicon discharge (e.g., the effect of the device aspect ratio [203], the helicon discharge sustained by the standing waves [207-209], the helicon plasmas near the lower hybrid frequency [211,212], helicon wave propagation in the toroidal geometry [213-215]).

In Secs. 11 and 12, the remaining critical issues, and several basic self-consistent fluid models of helicon discharge to solve them, are reviewed. There are five main issues to be further investigated: (1) The mode transition from the ICP to the helicon mode, (2) the density limit in helicon discharge, (3) the centrally peaked density profile, (4) instabilities, and (5) ion heating. These issues are closely related. The combination of the fluid models with the non-linear instability models and the neutral dynamics leads to thorough understanding of helicon discharge.

In Sec. 13, various applications of the helicon source are introduced. The compact helicon source using the permanent magnet $[6,256]$ is expected to be useful in the semiconductor industry. A next-generation electric thruster using the helicon source is now being developed for a spacecraft intended for deep-space travel [145, 257, 291]. The applications for nuclear fusion [298, 299, 301] and space plasma [7] are also being extensively studied.

The current helicon study should take the effects of the spatio-temporal non-linear dynamics and neutral dynamics into consideration, leading to a through understanding of helicon plasma production. The plasma transport and the equilibrium state in the helicon discharge should be discussed by including them. We believe that the attempt at a self-consistent model of helicon discharge will shed light on these issues, leading to the higher-efficiency helicon plasma production for future applications.

[1] R.W. Boswell, Plasma Phys. Control. Fusion 26, 1147 (1984).

[2] F.F. Chen, Plasma Phys. Control. Fusion 33, 339 (1991).

[3] S. Shinohara and H. Mizokoshi, Rev. Sci. Instrum. 77, 036108 (2006).

[4] S. Shinohara et al., Phys. Plasmas 16, 057104 (2009).

[5] S. Shinohara, J. Plasma Fusion Res. 78, 5 (2002).

[6] F.F. Chen, Plasma Sources Sci. Technol. 24, 014001 (2015).

[7] E.E. Scime et al., J. Plasma Phys. 81, 345810103 (2015).

[8] A.W. Trivelpiece and R.W. Gould, J. Appl. Phys. 30, 1784 (1959).

[9] Y. Mouzouris and J.E. Scharer, Phys. Plasmas 5, 4253 (1998).

[10] B.H. Park et al., IEEE Trans. Plasma Sci. 29. 502 (2001).

[11] K.P. Shamrai and S. Shinohara, Phys. Plasmas 8, 4659 (2001).

[12] D. Arnush, Phys. Plasmas 7, 3042 (2000).

[13] S. Isayama et al., Phys. Plasmas 23, 063513 (2016).

[14] D.D. Blackwell et al., Phys. Rev. Lett. 88, 145002 (2002).

[15] J.L. Kline et al., Phys. Rev. Lett. 88, 195002 (2002).

[16] J.L. Kline and E.E. Scime, Phys. Plasmas 10, 135 (2003).

[17] M. Krämer et al., Plasma Phys. Control. Fusion 49, A167 (2007).

[18] M. Light et al., Phys. Plasmas 8, 4675 (2001).

[19] G.R. Tynan et al., Phys. Plasmas 11, 5195 (2004).

[20] O. Grulke et al., Plasma Phys. Control. Fusion 49, B247 (2007).

[21] T. Yamada et al., Nature Phys. 4, 721 (2008).

[22] D. Bohm, The Characteristic of Electrical Discharges in Magnetic fields (Interscience Publishers, New York, 1949) p. 197.

[23] A. Fruchtman, J. Phys. D: Appl. Phys. 50, 473002 (2017).

[24] D.G. Miljak and F.F. Chen, Plasma Sources Sci. Technol. 7, 537 (1998)

[25] R.M. Magee et al., Phys. Plasmas 20, 123511 (2013).

[26] P. Aigrain, Proc. Conf. Semiconductor Physics 224 (1961).

[27] R. Bowers et al., Phys. Rev. Lett. 7, 339 (1961). 
[28] Y. Kanai, J. Appl. Phys. 2, 137 (1962).

[29] J.A. Lehane and P.C. Thonemann, Proc. Phys. Society 85, 301 (1965).

[30] J.P. Klosenberg et al., J. Fluid Mech. 21, 545 (1965).

[31] R.W. Boswell, Phys. Lett. A 33, 457 (1970).

[32] R.W. Boswell et al., Phy. Lett. A 91, 163 (1982).

[33] F.F. Chen, High Density Plasma Sources: Helicon Plasma Sources, ed. O.A. Popov (Park Ridge, NJ: Noyes) p. 60.

[34] P.K. Loewenhardt et al., Phys. Rev. Lett. 67, 2792 (1991).

[35] P. Zhu and R.W. Boswell, Phys. Fluids B: Plasma Phys. 3, 869 (1991).

[36] A.R. Ellingboe et al., Phys. Plasmas 2, 1807 (1995).

[37] A.W. Molvik et al., Phys. Rev. Lett. 79, 233 (1997).

[38] F.F. Chen and D.D. Blackwell, Phys. Rev. Lett. 82, 2677 (1999).

[39] K.P. Shamrai and V.B. Taranov, Plasma Phys. Control. Fusion 36, 1719 (1994).

[40] K.P. Shamrai and V.B. Taranov, Phys. Lett. A 204, 139 (1995).

[41] K.P. Shamrai and V.B. Taranov, Plasma Sources Sci. Technol. 5, 474 (1996).

[42] K.P. Shamrai et al., Plasma Phys. Control. Fusion 39, 505 (1997).

[43] K.P. Shamrai, Plasma Sources Sci. Technol. 7, 499 (1998).

[44] F.F. Chen, J. Vac. Sci. Thechnol. A 10, 1389 (1992).

[45] A. Komori et al., Phys. Fluids B: Plasma Phys. 3, 893 (1991).

[46] D. Curreli and F.F. Chen, Phys. Plasmas 18, 113501 (2011).

[47] A.R. Ellingboe and R.W. Boswel, Phys. Plasmas 3, 2797 (1996).

[48] A.W. Degeling et al., Phys. Plasmas 3, 2788 (1996).

[49] S. Rauf and M.J. Kushner, J. Appl. Phys. 82, 2805 (1997).

[50] S. Rauf and M.J. Kushner, IEEE Trans. Plasma Sci. 27, 1329 (1999).

[51] T. Kitajima et al., J. Appl. Phys. 84, 5928 (1998).

[52] J. Hopwood, Plasma Sources Sci. Technol. 1, 109 (1992).

[53] J.H. Keller, Plasma Sources Sci. Technol. 5, 166 (1996).

[54] A.W. Molvik et al., J. Vac. Sci. Technol. A 14, 984 (1996).

[55] A.J. Perry et al., J. Vac. Sci. Thechnol. B 9, 310 (1991).

[56] T. Shoji et al., Plasma Sources Sci. Technol. 2, 5 (1993).

[57] S. Shinohara et al., Plasma Phys. Control. Fusion 37, 1015 (1995).

[58] S. Shinohara et al., Thin Solid Films 316, 139 (1998).

[59] K.K. Chi et al., Plasma Sources Sci. Technol. 8, 421 (1999).

[60] K. Suzuki et al., Plasma Sources Sci. Technol. 7, 13 (1998).

[61] G. Cunge et al., Plasma Sources Sci. Technol. 8, 576 (1999).

[62] A.B. Altukhov et al., Phys. Plasmas 12, 022310 (2005).

[63] B. Lorenz et al., J. Vac. Sci. Thechnol. A 14, 623 (2005).

[64] Y.M. Aliev and M. Krämer, Phys. Plasmas 12, 072305 (2005).

[65] S. Shinohara and K. Yonekura, Plasma Phys. Control. Fusion 42, 41 (2000).

[66] Y. Sakawa et al., Phys. Plasmas 6, 4759 (1999).

[67] J.H. Kim and H.Y. Chang, Phys. Plasmas 3, 1462 (1996).

[68] P.A. Keiter et al., Phys. Plasmas 4, 2741 (1997).

[69] D.A. Schneider et al., Phys. Plasmas 6, 703 (1999).

[70] F.F. Chen, Phys. Fluids 4, 1448 (1961).

[71] N. D'Angelo and R.W. Motley, Plasma Phys. Fluids 6, 422 (1963)
[72] F.F. Chen, Phys. Fluids 10, 1647 (1967).

[73] H.W. Hendel, Phys. Fluids 11, 2426 (1968).

[74] T.K. Chu et al., Phys. Fluids 12, 203 (1969).

[75] R.F. Ellis et al., Plasma Phys. 22, 113 (1980).

[76] G.I. Kent et al., Plasma Phys. Fluids 12, 2140 (1969).

[77] D.L. Jassby, Phys. Rev. Lett. 25, 1567 (1970).

[78] F.W. Perkins and D.L. Jassby, Phys. Fluids 14, 102 (1971).

[79] D.L. Jassby, Phys. Fluids 15, 1590 (1972).

[80] F.C. Hoh, Phys. Fluids 6, 1184 (1963).

[81] A. Simon, Phys. Fluids 6, 382 (1963).

[82] M. Light et al., Plasma Sources Sci. Technol. 11, 273 (2002).

[83] F.F. Chen, Phys. Plasmas 3, 1783 (1996).

[84] R.W. Boswell and R.K. Porteous, Appl. Phys. Lett. 50, 1130 (1987).

[85] F.F. Chen and R.W. Boswell, IEEE Trans. Plasma Sci. 25, 1245 (1997).

[86] F.F. Chen et al., Plasma Sources Sci. Technol. 5, 173 (1996).

[87] F.F. Chen et al., Plasma Sources Sci. Technol. 3, 49 (1994).

[88] S. Shinohara et al., Plasma Phys. Control. Fusion 39, 1479 (1997).

[89] B. Davis, J. Plasma Phys. 4, 43 (1970).

[90] L. Spitzer, Physics of Fully Ionized Gases (Interscience Publishers, New York, 1962).

[91] R.W. Boswel, Aust. J. Phys. 25, 403 (1972).

[92] T. Lho et al., Phys. Plasmas 5, 3135 (1998).

[93] D. Arnush and F.F. Chen, Phys. Plasmas 5, 1239 (1998).

[94] S.H. Kim and Y.S. Hwang, Plasma Phys. Control. Fusion 50, 035007 (2008).

[95] F.F. Chen and D. Arnush, Phys. Plasmas 4, 3411 (1997).

[96] U. Kortshagen et al., J. Phys. D: Appl. Phys. 29, 1224 (1996).

[97] I.M. El-Fayoumi et al., J. Phys. D: Appl. Phys. 31, 3082 (1998).

[98] M.M. Turner and M.A. Liebeirman, Plasma Sources Sci. Technol. 8, 313 (1999).

[99] S. Shinohara, Jpn. J. Appl. Phys. 35, L731 (1996).

[100] S. Shinohara and K.P. Shamrai, Plasma Phys. Control. Fusion 42, 865 (2000).

[101] F.F. Chen and H. Torreblanca, Plasma Sources Sci. Technol. 16, 593 (2007).

[102] S.S. Kim et al., Phys. Plasmas 6, 2926 (1999).

[103] H.J. Lee et al., Plasma Sources Sci. Technol. 5, 383 (1996).

[104] O.V. Polomarov et al., Phys. Plasmas 12, 104505 (2005).

[105] O.V. Polomarov et al., IEEE Trans. Plasma Sci. 34, 767 (2006).

[106] A.U. Rehman and J.K. Lee, Phys. Plasmas 16, 083504 (2009).

[107] Y. Sakawa et al., Phys. Rev. Lett. 90, 105001 (2003).

[108] F.F. Chen, Physica Scripta T30, 14 (1990).

[109] T. Tanikawa and S. Shinohara, E-Proceedings of the 12th International Congress on Plasma Physics, Nice, 2004 (Association EURATOM-CAE, Cadarache, 2004), Paper No. P2-31.

[110] S. Shinohara, Trans. Fusion Sci. Technol. 63, 164 (2013).

[111] A. Fruchtman et al., Phys. Rev. Lett. 95, 115002 (2005).

[112] A. Fruchtman et al., Phys. Plasmas 15, 057102 (2008).

[113] A. Fruchtman, IEEE Trans. Plasma Sci. 36, 403 (2008).

[114] J.L. Raimbault et al., Phys. Plasmas 14, 013503 (2007).

[115] S.M. Rossnagel, J. Vac. Sci. Thechnol. A 8, 3113 (1990). 
[116] P. Chabert et al., J. Appl. Phys. 84, 161 (1998).

[117] V.M. Donnelly and M.V. Malyshev, Appl. Phys. Lett. 77, 2467 (2000).

[118] H. Abada et al., J. Appl. Phys. 92, 4223 (2002).

[119] M. Shimada et al., J. Appl. Phys. 103, 033304 (2008).

[120] G.R. Tynan, J. Appl. Phys. 86, 5356 (1999).

[121] S. Yun et al., Phys. Plasmas 7, 3448 (2000).

[122] T. Nakano et al., Appl. Phys. Lett. 58, 458 (1991).

[123] G.A. Hebner, J. Appl. Phys. 80, 2624 (1996).

[124] T. Hori et al., J. Vac. Sci. Technol. A 14, 144 (1996).

[125] J. Gilland et al., Plasma Sources Sci. Technol. 7, 416 (1996).

[126] I.D. Sudit and F.F. Chen, Plasma Sources Sci. Technol. 5, 43 (1996).

[127] A. Aanesland et al., Appl. Phys. Lett. 91, 121502 (2007).

[128] B. Clarenbach et al., Plasma Sources Sci. Technol. 12, 345 (2003).

[129] R.M. Magee et al., Phys. Plasmas 19, 123506 (2012).

[130] A.M. Keesee and E.E. Scime, Rev. Sci. Instrum. 77, 10F304 (2006).

[131] C.M. Denning et al., Phys. Plasmas 15, 072115 (2008).

[132] L. Liard et al., J. Phys. D: Appl. Phys. 40, 5192 (2007).

[133] L. Liard et al., J. Phys. D: Appl. Phys. 45, 235201 (2012).

[134] A. Fruchtman, Plasma Sources Sci. Technol. 17, 024016 (2008).

[135] L. Tonks and I. Langmuir, Phys. Rev. 34, 876 (1929).

[136] W. Schottky, Phys. Z 25, 635 (1924).

[137] V. Godyak, Soviet Radiofrequency Discharge Research (Delphic Associates, Fall Church, VA, 1986).

[138] A. Fruchtman and J. -M. Rax, Phys. Plasmas 17, 043502 (2010).

[139] L. Liard et al., Phys. Plasmas 16, 053507 (2009).

[140] A. Fruchtman, Plasma Sources Sci. Technol. 18, 025033 (2009).

[141] A. Fruchtman, Phys. Plasmas 17, 023502 (2010).

[142] A. Fruchtman and S. Shinohara, Phys. Plasmas 24, 103523 (2017).

[143] B.R. Roberson et al., Phys. Plasmas 18, 053505 (2011).

[144] K. Takahashi et al., Phys. Rev. Lett. 110, 195003 (2013).

[145] K. Takahashi et al., Plasma Sources Sci. Technol. 25, 055011 (2016).

[146] K. Takahashi and A. Ando, Phys. Rev. Lett. 118, 225002 (2017).

[147] E.E. Scime et al., Phys. Plasmas 7, 2157 (2000).

[148] R.L. Stenzel and J.M. Urrutia, Phys. Plasmas 7, 4450 (2000).

[149] C.S. Corr and R.W. Boswell, Phys. Plasmas 14, 122503 (2007).

[150] S. Shinohara et al., Plasma Sources Sci. Technol. 19, 034018 (2010).

[151] S. Shinohara et al., Phys. Plasmas 23, 122108 (2016).

[152] F.F. Chen and D. Curreli, Phys. Plasmas 20, 057102 (2013).

[153] J.D. Evans et al., Phys. Rev. Lett. 86, 5502 (2001).

[154] R.A. Demirkhanov et al., Soviet Phys. 19, 791 (1964).

[155] M.J. Kofoid and J.M. Dawson, Phys. Rev. Lett. 17, 1086 (1966).

[156] E.S. Weibel, Plasma Phys. Fluids 10, 741 (1967).

[157] B. Joye and H. Schnneider, Helv. Phys. Acta 51, 804 (1978).

[158] Y.S. Sayakov, Helv. Phys. Acta 52, 288 (1979).

[159] V.I. Kolobov and V.A. Godyak, IEEE Trans. Plasma Sci. 23, 503 (1995).

[160] V.I. Kolobov and D.J. Economou, Plasma Sources Sci.
Technol. 6, R1 (1999).

[161] V.A. Godyak et al., Phys. Rev. Lett. 83, 1610 (1999).

[162] V.A. Godyak et al., Plasma Sources Sci. Technol. 9, 541 (2000).

[163] V.A. Godyak et al., Plasma Sources Sci. Technol. 10, 459 (2001).

[164] A.I. Smolyakov et al., Phys. Plasmas 10, 2108 (2003).

[165] V.A. Godyak and V. Kolobov, Phys. Rev. Lett. 79, 4589 (1997).

[166] Yu.O. Tyshetskiy et al., Plasma Sources Sci. Technol. 11, 203 (2002).

[167] F.F. Chen, IEEE Trans. Plasma Sci. 34, 718 (2006).

[168] D. Curreli and F.F. Chen, Plasma Sources Sci. Technol. 23, 064001 (2014).

[169] S.I. Braginskii, Transport properties in a plasma (Consultants Bureau, New York, 1965).

[170] A. Simon, Phys. Rev. 98, 317 (1955).

[171] A. Simon and R.V. Neidigh, Diffusion of ions in a plasma across a magnetic field (Oak Ridge National Laboratory, 1955).

[172] T. Lafleur and R.W. Boswell, Phys. Plasmas 19, 053505 (2012).

[173] F.C. Hoh and B. Lehnert, Phys. Fluids 3, 600 (1960).

[174] T.K. Allen et al., Phys. Rev. Lett. 5, 409 (1960).

[175] J.F. Bonnal et al., Phys. Rev. Lett. 6, 665 (1961).

[176] B.B. Kadomtsev and A.V. Nedospasov, J. Nucl. Energy Part C 1, 230 (1960).

[177] N. D'Angelo and N. Rynn, Phys. Fluids 4, 1303 (1961).

[178] N. D'Angelo and N. Rynn, Phys. Fluids 4, 275 (1961).

[179] F.F. Chen, J. Nucl. Energy Part C 7, 399 (1965).

[180] W. Horton, Rev. Mod. Phys. 71, 735 (1999).

[181] N. Rynn and N. D’Angelo, Rev. Sci. Instrum. 31, 1326 (1960).

[182] S.C. Prager et al., Phys. Rev. Lett. 33, 692 (1974).

[183] J. Slough et al., Phys. Rev. Lett. 47, 1057 (1981).

[184] R. Scarmozzino et al., Phys. Rev. Lett. 57, 1729 (1986).

[185] A.K. Sen et al., Phys. Rev. Lett. 66, 429 (1991).

[186] C. Holland et al., Phys. Rev. Lett. 96, 195002 (2006).

[187] S.J. Camargo et al., Phys. Plasmas 2, 48 (1995).

[188] A. Hasegawa and M. Wakatani, Phys. Rev. Lett. 59, 1581 (1987).

[189] P. Manz et al., Plasma Phys. Control. Fusion 53, 095001 (2011).

[190] G.Y. Antar et al., Phys. Plasmas 14, 022301 (2007).

[191] C.M. Franck et al., Phys. Plasmas 10, 323 (2003).

[192] O. Grulke et al., Phys. Plasmas 6, 788 (1999).

[193] C. Schröderl et al., Phys. Plasmas 11, 4249 (2004).

[194] C. Schröderl et al., Phys. Plasmas 12, 42103 (2005).

[195] O. Grulke et al., Contrib. Plasma Phys. 45, 385 (2005).

[196] T. Windisch et al., Phys. Plasmas 13, 122303 (2006).

[197] F. Brochard et al., Phys. Plasmas 13, 122305 (2006).

[198] K. Terasaka et al., Plasma Fusion Res. 2, 031 (2007).

[199] A.I. Akhiezer et al., Phys. Lett. A 245, 117 (1998).

[200] J.L. Kline et al., Phys. Plasmas 10, 2127 (2003).

[201] V.S. Mikhailenko et al., Phys. Plasmas 10, 2247 (2003).

[202] S. Shinohara and T. Soejima, Plasma Phys. Control. Fusion 40, 2081 (1998).

[203] T. Motomura et al., Phys. Plasmas 19, 043504 (2012).

[204] S. Shinohara et al., Surf. Coat. Technol. 112, 20 (1999).

[205] S. Shinohara et al., Jpn. J. Appl. Phys. 38, 4321 (1999).

[206] S. Shinohara and T. Tanikawa, Phys. Plasmas 12, 044502 (2005).

[207] M. Nisoa et al., Jpn. J. Appl. Phys. 38, L777 (1999).

[208] M. Nisoa et al., Jpn. J. Appl. Phys. 39, L429 (2000). 
[209] M. Nisoa et al., Jpn. J. Appl. Phys. 40, 3396 (2001).

[210] A. Fukuyama and Y. Ichida, Proc. 1996 Int. Conf. Plasma Physics, Nagoya 1996 (Japan Society of Plasma Science and Nuclear Fusion Research, Nagoya) 2, 1342 (1997).

[211] S.M. Yun, J. Vac. Sci. Technol. A 15, 673 (1997).

[212] S.M. Yun and H.Y. Chang, Phys. Lett. A 248, 400 (1998).

[213] P.K. Loewenhardt et al., Plasma Phys. Control. Fusion 37, 229 (1995).

[214] B.C. Zhang et al., Plasma Phys. 2, 803 (1995).

[215] L. Chang et al., Phys. Plasmas 19, 083511 (2012).

[216] D.D. Blackwell et al., Plasma Sources Sci. Technol. 21, 055033 (2012).

[217] C.M. Franck et al., Plasma Sources Sci. Technol. 14, 226 (2005).

[218] C.S. Corr et al., Phys. Plasmas 11, 4596 (2004).

[219] A. Ganguli et al., Phys. Plasmas 14, 113503 (2007).

[220] A. Ganguli et al., Plasma Sources Sci. Technol. 20, 015201 (2011).

[221] A. Ganguli et al., Phys. Plasmas 20, 013510 (2013).

[222] B. Breizman et al., Phys. Rev. Lett. 84, 3863 (2000).

[223] M.I. Panevsky and R.D. Bengtson, Phys. Plasmas 11, 4196 (2004).

[224] G. Chen et al., Phys. Plasmas 13, 123507 (2006).

[225] C.A. Lee et al., Phys. Plasmas 18, 013501 (2011).

[226] M.A. Lieberman and A.J. Lichtenberg, Principles of Plasma Discharges and Materials Processing: Second Edition (Wiley Interscience, 2005).

[227] J.D. Callen, Fundamentals of Plasma Physics Chapter 2: Coulomb Collisions (Public homepage of J.D. Callen, UW-Madison).

[228] R.L. Kinder and M.J. Kushner, J. Vac. Sci. Technol. A 19, 76 (2001).

[229] R.L. Kinder and M.J. Kushner, J. Appl. Phys. 90, 3699 (2001).

[230] G.R. Tynan et al., J. Vac. Sci. Technol. A 15, 2885 (1997).

[231] R.L. Kinder et al., Plasma Sources Sci. Technol. 12, 561 (2003).

[232] A.W. Degeling et al., Phys. Plasmas 6, 1641 (1999).

[233] M.J. Grapperhaus and M.J. Kushner, J. Appl. Phys. 81, 569 (1997)

[234] D. Bose et al., IEEE Trans. Plasma Sci. 31, 464 (2003).

[235] S. Cho and M.A. Lieberman, Phys. Plasmas 10, 882 (2003).

[236] G. Chen, Phys. A self-consistent model of helicon discharge ( $\mathrm{PhD}$ thesis, The University of Texas at Austin, 2008).

[237] S. Isayama et al., Proc. in 1st Asia Pacific Conf., Chengdu, 63513 (2017).

[238] G.N. Kervalishvili et al., Plasma Phys. 46, 739 (2006).

[239] N. Kasuya et al., J. Plasma Phys. 72, 957 (2006).

[240] N. Kasuya et al., J. Phys. Soc. Jpn. 76, 044501 (2007).

[241] N. Kasuya et al., Phys. Plasmas 15, 052302 (2008).

[242] B.D. Scott, Plasma Phys. Control. Fusion 45, A385 (2003).

[243] L. Chang et al., Phys. Plasmas 18, 042106 (2011).

[244] G.A. Bird, Molecular gas dynamics and the direct simulation of gas flows (Oxford Engineering Science Series 47, 1996).

[245] M.J. Hartig and M.J. Kushner, Appl. Phys. Lett. 62, 1594 (1993).

[246] M.D. Kilgore et al., J. Vac. Sci. Technol. B 12, 494 (1994).

[247] V.V. Serikov et al., IEEE Trans. Plasma Sci. 27, 1389 (1999).
[248] K. Nanbu, IEEE Trans. Plasma Sci. 28, 971 (2000).

[249] M. Shimada et al., J. Vac. Sci. Technol. A 24, 1878 (2006).

[250] M. Shimada et al., Plasma Sources Sci. Technol. 16, 193 (2007).

[251] K. Aoki et al., Toshiba Review 55, 17 (2000).

[252] N. Benjamin et al., Proc. SPIE 95, 1392 (1991).

[253] I. Tepermeister et al., J. Vac. Sci. Technol. B 12, 2310 (1994).

[254] I. Tepermeister et al., J. Vac. Sci. Technol. B 12, 2322 (1994).

[255] G.E. Gibson et al., J. Vac. Sci. Technol. B 12, 2333 (1994).

[256] F.F. Chen, Phys. Plasmas 19, 093509 (2012).

[257] F.R.C. Diaz, The VASIMR Rocket (Scientific American, November, 2000).

[258] B.W. Longmier et al., Proc. on 32nd International Electric Propulsion Conference, 156 (2011).

[259] B.W. Longmier et al., Plasma Sources Sci. Technol. 20, 015007 (2011).

[260] L.D. Cassady et al., Proc. 46th AIAA/ASME/SAE/ASEE Joint Propulsion Conference, Nashville, TN, 6772 (2010).

[261] E.A. Bering et al., Proc. 49th AIAA Aerospace Sciences Meeting, Orlando, FL, 1071 (2011).

[262] B.W. Longmier et al., Proc. 48th AIAA/ASME/SAE/ ASEE Joint Propulsion Conference, Atlanta, Georgia, 3930 (2012).

[263] B.W. Longmier et al., J. Prop. and Power 30, 123 (2014).

[264] K. Takahashi et al., Phys. Plasmas 15, 074505 (2008).

[265] K. Takahashi et al., Appl. Phys. Lett. 94, 191503 (2009).

[266] K. Takahashi et al., Phys. Rev. Lett. 107, 235001 (2011).

[267] K. Takahashi et al., Appl. Phys. Lett. 98, 141503 (2011).

[268] K. Takahashi et al., J. Phys. D: Appl. Phys. 46, 352001 (2013).

[269] K. Takahashi et al., Phys. Rev. Lett. 114, 195001 (2015).

[270] K. Takahashi et al., Appl. Phys. Lett. 109, 194101 (2016).

[271] K. Takahashi et al., Appl. Phys. Lett. 108, 074103 (2016).

[272] C. Charles and R.W. Boswell, Appl. Phys. Lett. 82, 1356 (2003).

[273] C. Charles and R.W. Boswell, Phys. Plasmas 11, 1706 (2004).

[274] C. Charles et al., Appl. Phys. Lett. 89, 261503 (2006).

[275] C. Charles, J. Phys. D: Appl. Phys. 42, 163001 (2009).

[276] A. Fruchtman, Phys. Rev. Lett. 96, 065002 (2006).

[277] E. Ahedo and M. Merino, Phys. Plasmas 17, 073501 (2010).

[278] E. Ahedo and J. Navarro-Cavallé, Phys. Plasmas 20, 043512 (2013).

[279] S. Pottinger et al., J. Phys. D: Appl. Phys. 44, 235201 (2011).

[280] T. Nakamura et al., World Academy of Science, Engineering and Technology 71, 1498 (2012).

[281] L.T. Williams and W.L. Walker, J. Propulsion Power 29, 520 (2013).

[282] A. Shabshelowitz and A.D. Gallimore, J. Propulsion Power 29, 919 (2013).

[283] Y. Takao and K. Takahashi, Phys. Plasmas 22, 113509 (2015).

[284] K. Toki et al., Proc. 29th International Electric Propulsion Conference, 03-0168 (2003).

[285] T. Matsuoka et al., Plasma Fusion Res. 6, 2406103 (2011).

[286] S. Satoh et al., Proc. 42th AIAA Plasmadynamics and Lasers Conference 4008 (2011). 
[287] T. Matsuoka et al., Jpn. J. Appl. Phys. 51, 096201 (2012).

[288] H. Nishida et al., Appl. Plasma Technol. 5, 67 (2012).

[289] S. Shinohara et al., Proc. 19th IEEE Pulsed Power Conference 6627580 (2013).

[290] F. Otsuka et al., Plasma Fusion Res. 8, 1406012 (2013).

[291] S. Shinohara et al., IEEE Trans. Plasma Sci. 42, 1245 (2014).

[292] T. Ishii et al., Proc. 12th Asia Pacific Physics Conference, 015047 (2014).

[293] S. Otsuka et al., Plasma Fusion Res. 10, 3401026 (2015).

[294] F. Otsuka et al., Earth, Planets Space 67, 85 (2015).
[295] N. Ohnishi et al., Jpn. J. Appl. Phys. 55, 01602 (2016).

[296] D.D. Blackwell et al., J. Nucl. Fusion 25, 1485 (1985).

[297] S.M. Hamberger et al., Fusion Technol. 17, 123 (1990).

[298] M.G. Shats et al., Phys. Rev. Lett. 77, 4190 (1996).

[299] M.G. Shats et al., Phys. Plasmas 4, 3629 (1997).

[300] M.G. Shats et al., Phys. Plasmas 5, 2390 (1998).

[301] S.K.P. Tripathi and D. Bora, Phys. Plasmas 8, 697 (2001).

[302] S.K.P. Tripathi et al., J. Phys. 56, 551 (2001).

[303] S. Houshmandyar and E.E. Scime, Phys. Plasmas 18, 112111 (2011). 